\title{
Kinematics of the Circumgalactic Medium of a $z=0.77$ Galaxy from Mg II Tomography
}

\author{
Kris Mortensen $^{1}\left(\mathbb{1}\right.$, Keerthi Vasan G. C. ${ }^{1}$, Tucker Jones ${ }^{1}$ (D), Claude-André Faucher-Giguère ${ }^{2}$ (1), Ryan L. Sanders ${ }^{1}$ (1) \\ Richard S. Ellis $^{3}$ (D), Nicha Leethochawalit ${ }^{4,5}$ (D), and Daniel P. Stark ${ }^{6}$ \\ ${ }^{1}$ Department of Physics, University of California, Davis, 1 Shields Avenue, Davis, CA 95616, USA; kpmort@ucdavis.edu \\ ${ }^{2}$ Department of Physics and Astronomy, Northwestern University, 2145 Sheridan Road, Evanston, IL 60201, USA \\ ${ }^{3}$ Department of Physics and Astronomy, University College London, Gower Street, London WC1E 6BT, UK \\ ${ }^{4}$ School of Physics, The University of Melbourne, Parkville, VIC 3010, Australia \\ ${ }^{5}$ ARC Centre of Excellence for All Sky Astrophysics in 3 Dimensions (ASTRO 3D), Australia \\ ${ }^{6}$ Steward Observatory, University of Arizona, 933 North Cherry Avenue, Tucson, AZ 85721, USA \\ Received 2020 May 28; revised 2021 February 27; accepted 2021 April 19; published 2021 June 18
}

\begin{abstract}
Galaxy evolution is thought to be driven in large part by the flow of gas between galaxies and the circumgalactic medium (CGM), a halo of metal-enriched gas extending out to $\gtrsim 100 \mathrm{kpc}$ from each galaxy. Studying the spatial structure of the CGM holds promise for understanding these gas flow mechanisms; however, the common method of using background quasar sight lines provides minimal spatial information. Recent works have shown the utility of extended background sources such as giant gravitationally lensed arcs. Using background lensed arcs from the CSWA 38 lens system, we continuously probed, at a resolution element of about $15 \mathrm{kpc}^{2}$, the spatial and kinematic distribution of $\mathrm{Mg}$ II absorption in a star-forming galaxy at $z=0.77$ (stellar mass $\approx 10^{9.7} M_{\odot}$, star formation rate $\approx 10 M_{\odot} \mathrm{yr}^{-1}$ ) at impact parameters $D \simeq 5-30 \mathrm{kpc}$. Our results present an anisotropic, optically thick medium whose absorption strength decreases with increasing impact parameter, in agreement with the statistics toward quasars and other gravitational arcs. Furthermore, we find generally low line-of-sight velocities in comparison to the relatively high velocity dispersion in the $\mathrm{Mg}$ II gas (with typical $\sigma \approx 50 \mathrm{~km} \mathrm{~s}^{-1}$ ). While the galaxy itself exhibits a clear outflow (with $\mathrm{Mg}$ II velocities up to $\sim 500 \mathrm{~km} \mathrm{~s}^{-1}$ ) in the down-the-barrel spectrum, the outflow component is subdominant and only weakly detected at larger impact parameters probed by the background arcs. Our results provide evidence of mainly dispersion-supported, metal-enriched gas recycling through the CGM.
\end{abstract}

Unified Astronomy Thesaurus concepts: Circumgalactic medium (1879); Interstellar absorption (831); Galaxy formation (595); Galaxy fountains (596); Galaxy winds (626)

\section{Introduction}

Observing the distribution and kinematics of gas within galaxies is a major challenge in understanding galactic evolution. Much of the activity that drives a galaxy's evolution occurs in the circumgalactic medium (CGM; e.g., AnglésAlcázar et al. 2017; Hafen et al. 2020), a region between a galaxy and the intergalactic medium (Rudie et al. 2012; Shull 2014). The rate of gas accretion from the CGM is the primary driver of the star formation rate (SFR) in galaxies (e.g., Dekel et al. 2009; van de Voort et al. 2011). Accretion of CGM gas with modest metallicity also explains the relative paucity of low-metallicity stars within the disk (van den Bergh 1962; Sommer-Larsen 1991; Woolf \& West 2012), as well as the existence of high-column but low-metallicity IGM absorbers (e.g., Lehner et al. 2013; Hafen et al. 2017).

The CGM regions typically have been probed at $10-100 \mathrm{kpc}$ scales through absorption seen in the spectra of background quasars (e.g., Nielsen et al. 2013b; Chen 2017; Prochaska et al. 2017; Tumlinson et al. 2017) and galaxies (e.g., Steidel et al. 2010; Diamond-Stanic et al. 2016; Rubin et al. 2018b). These techniques have yielded critical observational constraints and evidence toward the relationship between the CGM and galaxy properties; however, the data gathered from such probes rarely provide conclusions on the spatial structure within each CGM. Unless a galaxy has the rare privilege of multiple sight lines piercing through its CGM (e.g., Lehner et al. 2020), spatial information requires either stacking the spectra of background sources (e.g., Steidel et al. 2010; Bordoloi et al. 2011; Rubin et al. 2018a, 2018c) or averaging ensembles of absorber properties (Chen et al. 2010; Nielsen et al. 2013a). While such statistical studies provide important information regarding the average CGM profile around different galaxy populations, they provide only a crude view of the CGM structure around individual galaxies. Some effort has been made to probe the CGM of intervening galaxies through the use of multiple sight lines, especially multiply imaged lensed quasars (Smette et al. 1992; Lopez et al. 1999, 2005, 2007; Rauch et al. 2001; Ellison et al. 2004; Chen et al. 2014; Zahedy et al. 2016; Rubin et al. 2018c; Zabl et al. 2020). While these quasars help to resolve kiloparsec (kpc) scales within the CGM, the scarcity of such observed objects results in small samples and a limited sampling relative to the overall CGM areal extent. However, recent observations of extended gravitationally lensed arcs (Lopez et al. 2018, 2020) provide enhanced spatial sampling of the CGM, probing gaseous halos in individual galaxies on scales of $1-100 \mathrm{kpc}$ without potential biases or loss of information introduced by stacking techniques.

In this paper, we probe the the spatial and kinematic distribution of $\mathrm{Mg}$ II in the CGM of a $z=0.77$ galaxy based on spatially resolved spectroscopy of CSWA 38, one of the gravitational lens systems cataloged in the Cambridge And Sloan Survey Of Wide ARcs in the skY (with target names abbreviated as CSWA; Belokurov et al. 2007, 2009). The system consists of a galaxy cluster at $z=0.43$ with two luminous giant arcs at $z \approx 2.92$ and multiple other moderately magnified background sources (Figure 1; Koester et al. 2010; Bayliss et al. 2011). The subject of this study is a $z=0.77$ galaxy that lies between the two giant arcs. Moderateresolution spectroscopy revealed prominent $\mathrm{Mg}$ II and Fe II 

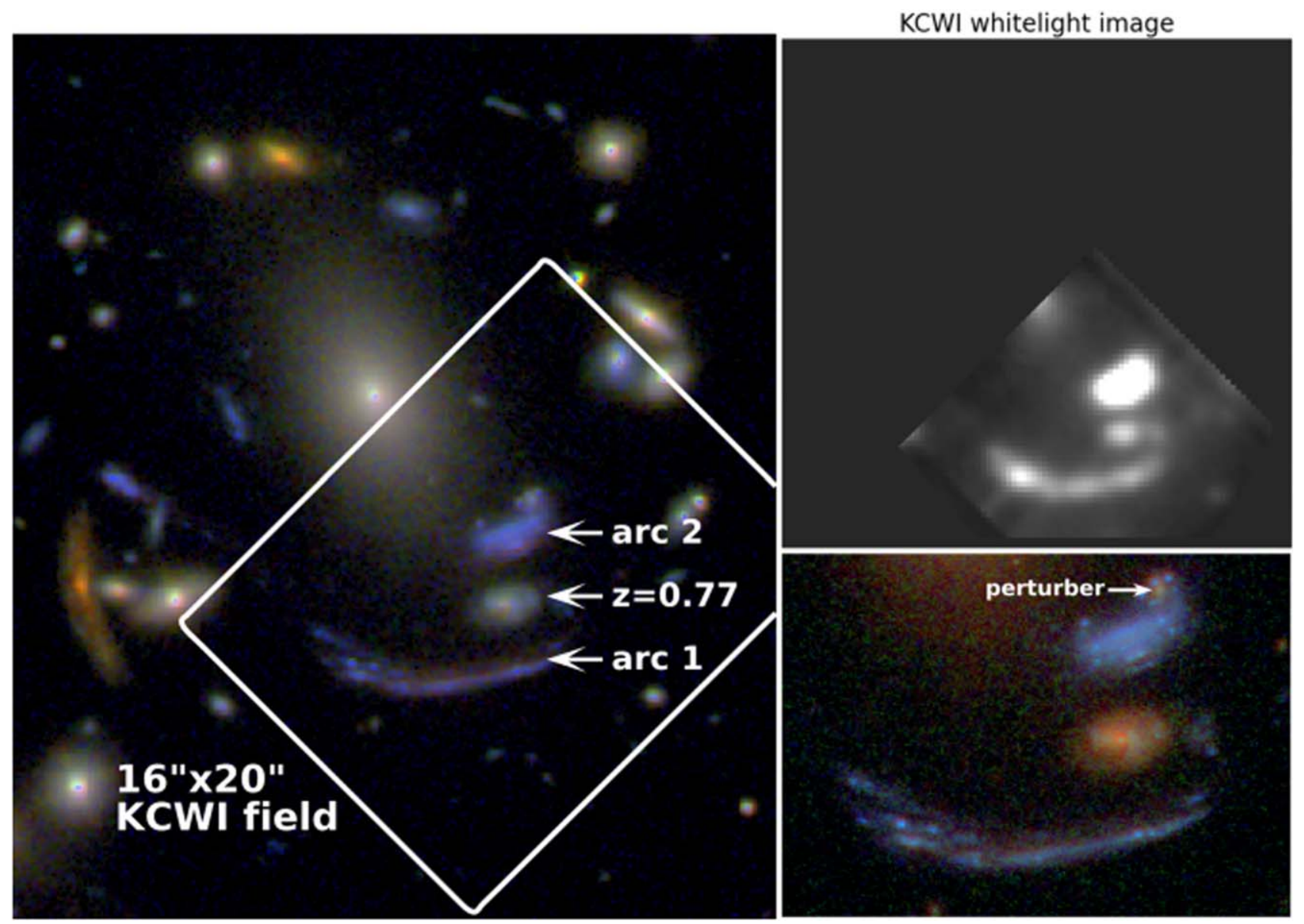

Figure 1. Color HST images of the CSWA 38 lens system. North is up, and east is to the left. Left: F160W/F110W/F814W color image with the KCWI field of view shown for scale (oriented at a sky PA of $135^{\circ}$ ). The $z=2.92$ arcs and $z=0.77 \mathrm{Mg}$ II absorption host are labeled. Top right: KCWI white-light image centered on the absorption host and background arcs. Bottom right: F110W/F814W/F606W image zoomed in on the $z=0.77$ target and background arcs. A perturber galaxy is evident in HST imaging near arc 2, creating multiple images of region C (see Figure 2), and its mass profile is included in the lens model.

absorption at $z=0.77$ in the background arc spectra (Jones et al. 2018), making this system an ideal candidate for the CGM absorption tomography presented herein.

The paper is structured as follows. In Sections 2 and 3, we describe spectroscopic observations of the lensing system, as well as the lens model used to demagnify the absorber galaxy and calculate impact parameters in the absorber plane. Sections 4 and 5 present the main analysis of the absorber galaxy properties and the line strength and kinematics of the Mg II gas. We discuss our results in Section 6 and present our summary and conclusions in Section 7. Throughout this paper, we adopt a flat $\Lambda \mathrm{CDM}$ cosmology with $H_{0}=70 \mathrm{~km} \mathrm{~s}^{-1} \mathrm{Mpc}^{-1}, \Omega_{\mathrm{m}}=0.26$, and $\Omega_{\Lambda}=$ 0.74 .

\section{Spectroscopic Data}

The system CSWA 38 was observed with the Keck Cosmic Web Imager (KCWI; Morrissey et al. 2018) on 4 nights during three separate observing runs. Two orthogonal sky position angles (PA) were used with comparable depth in each. We observed at a PA of $135^{\circ}$ on 2018 June 17 (90 minutes on source) and 2020 June 20 (100 minutes) and $45^{\circ}$ on 2019 June 2 (60 minutes) and 2020 June 19 (97 minutes). The total onsource exposure time is thus 347 minutes or $5.8 \mathrm{hr}$. Two exposures (40 minutes on source) taken on 2020 June 19 were offset to cover the eastern counterimage of arc 2, while the remaining exposures were approximately centered on the $z=0.77$ absorber galaxy and two bright arcs (see Figures 1 and 2).

Individual exposure times were 600-1200 s. Conditions ranged from clear to $0.5 \mathrm{mag}$ of cloud extinction, with 0 "! $8-1$ "! 1 seeing. The KCWI was configured with the medium slicer $(0$ "! 7 slit width), BL grating, and central wavelength $\lambda_{c}=5150 \AA$ with the blue blocking filter retracted. This provides wavelength coverage from approximately 4000 to $6300 \AA$. This range includes the $\mathrm{Mg}$ II $\lambda \lambda 2796,2802$ doublet redshifted to $\sim 4950 \AA$ at $z=0.77$ and several Fe II transitions at rest frame 2344-2600 ̊. From arc lamp exposures, we measure an approximately constant spectral FWHM $=2.40 \AA$ with $<5 \%$ variation across the full wavelength range (e.g., $R=2060$ at $\lambda=4950 \AA$ ).

Data were reduced using the KCWI data reduction pipeline (KDERP) version 1.0.2. It performs instrument signature removal (bias, dark current, scattered light, and flat-fielding), sky subtraction, wavelength calibration, and spatial rectification, including a correction for differential atmospheric refraction. Output data cubes have 0 ". $68 \times 0$ ". 29 spatial pixels. Observations of the standard stars HZ43, BD+25d3941, BD+26d2608, and G93-48 were taken on the same nights and used for flux calibration of the 2018, 2019, and 2020 data, respectively. The pipeline-reduced data cubes have a nonzero residual background with a spatial gradient that affects measurements of absorption equivalent width if not properly corrected. We model this residual 


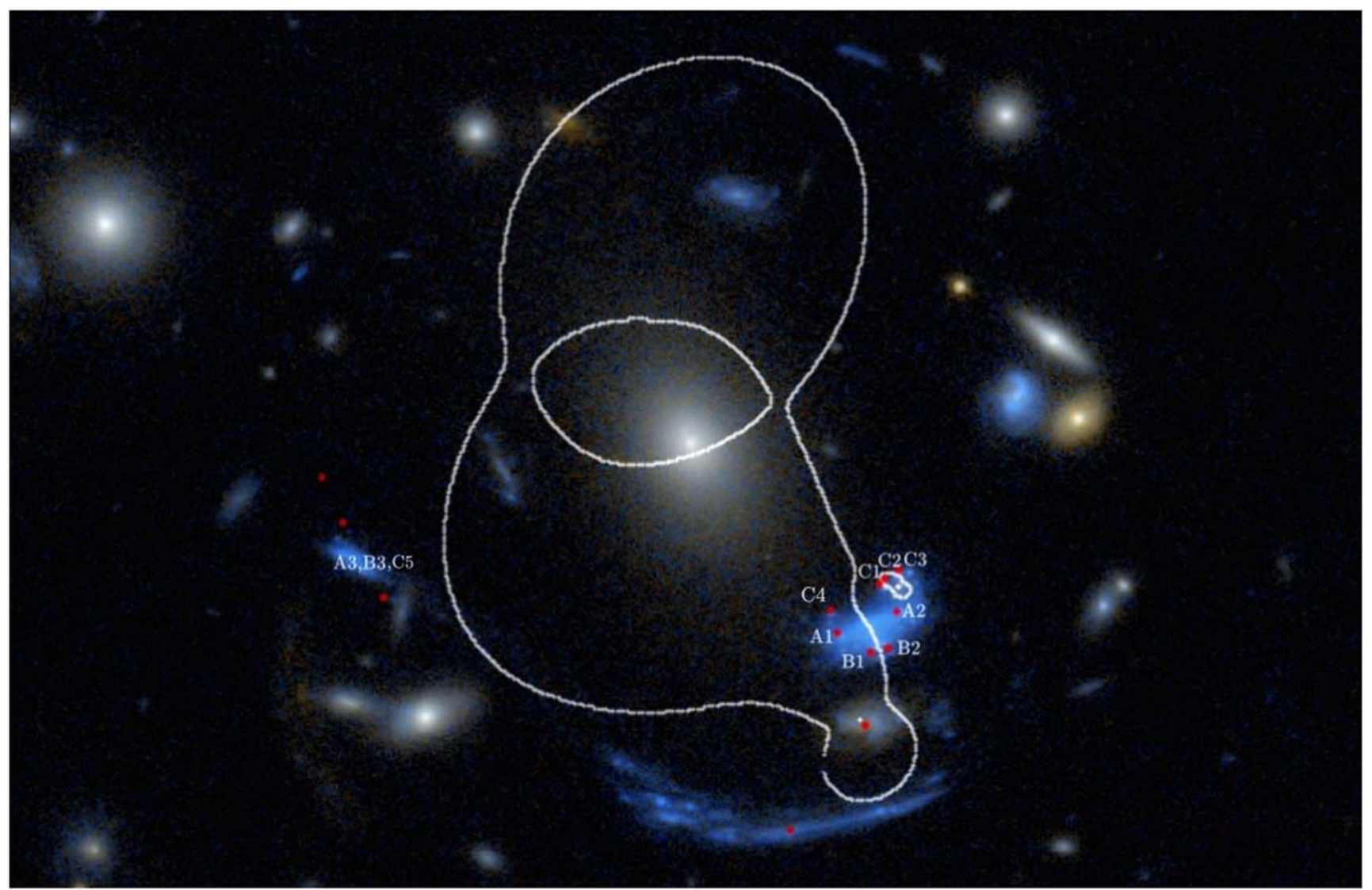

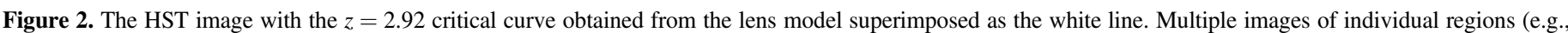

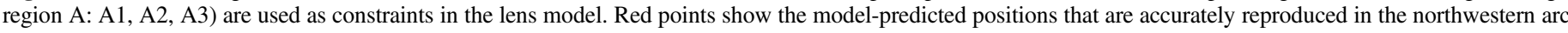

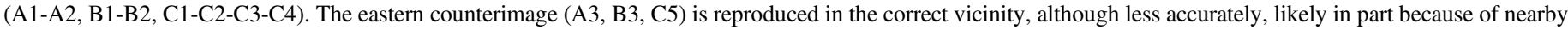
substructure that is not included in the model.

structure with a two-dimensional first-order polynomial (i.e., a plane) fit to blank sky regions in a pseudo-image generated by taking the median flux in each spaxel over the wavelength range around the $\mathrm{Mg}$ II absorption ( $\lambda=4834-5084 \AA$ ). We subtract this fit from each wavelength slice of the data cube. This approach is similar to the correction described by Burchett et al. (2021), and we find that it adequately removes the residual spatial background structure at the wavelengths of interest.

The sky-corrected data cubes from individual exposures were resampled to a common grid and combined with a weighted mean. The resulting data cube has $0 . " 3$ spatial pixels, adequately sampling both the native pixel size and the seeing. This final data cube is used for all subsequent analysis. A "white-light" image of this data cube, created by summing all pixels in the wavelength direction, is shown in Figure 1.

\section{Gravitational Lens Model}

In order to study the impact parameter of the CGM probed by the arcs, we must account for gravitational lensing of the region around the $\mathrm{Mg}$ II absorber galaxy. In this section, we describe the adopted lens model that sufficiently reproduces all of the observational constraints. We model the lens as a combination of a galaxy cluster-scale dark matter halo plus individual galaxies, considering only those galaxies that significantly affect the lens model in the vicinity of the Mg II absorber galaxy and bright arcs (Figure 1). We ignore the perturbers that are farther away, since they have a negligible effect on the results of this work and we
Table 1

R.A. and Decl. Positions of Objects Included in the Lens Model

\begin{tabular}{lcc}
\hline \hline Object & $\alpha$ & $\delta$ \\
\hline cD+cluster $(z=0.43)$ & $12: 26: 51.7$ & $+21: 52: 25.4$ \\
Perturber $(z=0.43)$ & $12: 26: 51.2574$ & $+21: 52: 21.214$ \\
Absorber $(z=0.77)$ & $12: 26: 51.3325$ & $+21: 52: 17.154$ \\
\hline
\end{tabular}

Note. Galaxy centroid positions are determined from HST optical (F606W) images. The perturber redshift is unknown and assumed to be at the same $z=0.43$ as the cluster.

lack suitable constraints on the lensing potential for regions beyond the bright arcs.

The lens model is constructed using the Glafic (Oguri 2010) package, with the following mass components (listed in Tables 1 and 2). The cluster mass distribution is modeled as a NavarroFrenk-White (NFW) profile (Navarro et al. 1997) at $z=0.43$, with the central dominant (cD) galaxy modeled as a singular isothermal ellipsoid (SIE). Another SIE profile at $z=0.43$ is added to model the effect of the perturber galaxy seen to the north of arc 2 (Figure 1). Although we are unable to confirm spectroscopically, the perturber is photometrically consistent with the cluster redshift, having similar colors to the cluster red sequence galaxies (e.g., HST F606W-F814W $\approx 1.1$ ). We note that its precise redshift does not affect the results of this work. The absorber galaxy at $z=0.77$ is modeled as a singular isothermal sphere (SIS). The SIS is scaled and treated as existing in the 

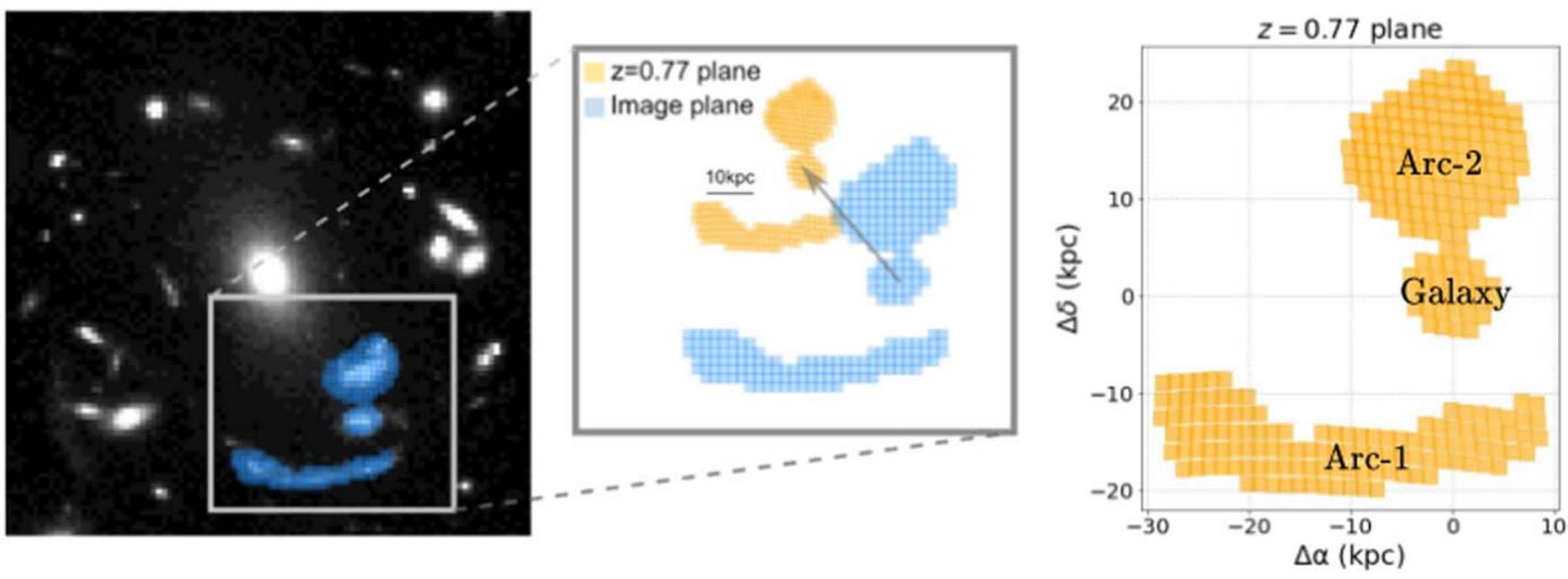

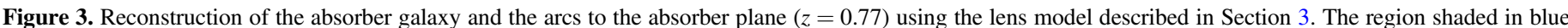

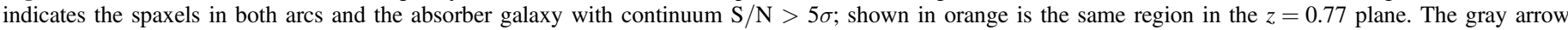

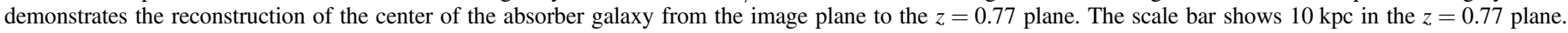

Table 2

Best-fit Parameters of the Lens Model

\begin{tabular}{|c|c|c|c|c|c|c|c|c|c|c|}
\hline $\begin{array}{l}\text { Component } \\
\text { and Profile }\end{array}$ & $\begin{array}{c}\sigma \\
\text { or } M\end{array}$ & $\begin{array}{c}\sigma_{\text {prior }} \\
\text { or } M_{\text {prior }}\end{array}$ & $x$ & $\begin{array}{c}x_{\text {prior }} \\
(\operatorname{arcsec})\end{array}$ & $y$ & $\begin{array}{c}y_{\text {prior }} \\
(\operatorname{arcsec})\end{array}$ & $e$ & $e_{\text {prior }}$ & PA & $\mathrm{PA}_{\text {prior }}$ \\
\hline cD-SIE & $490\left(\mathrm{~km} \mathrm{~s}^{-1}\right)$ & $\mathrm{G}(430,100)$ & 0.37 & $\mathrm{G}(0,0.2)$ & -0.34 & $\mathrm{G}(0,0.2)$ & 0.41 & $\mathrm{U}(0.2,0.5)$ & 22 & $\overline{\mathrm{U}(10,30)}$ \\
\hline Cluster-NFW & $3.0 \times 10^{14}\left(h^{-1} M_{\odot}\right)$ & $\mathrm{G}(3 \mathrm{e}+14,2 \mathrm{e}+14)$ & 1.25 & $\mathrm{U}(-3,3)$ & 1.94 & $\mathrm{U}(-3,3)$ & 0.39 & $\mathrm{U}(0.2,0.5)$ & 147 & $\ldots$ \\
\hline Perturber-SIE & $59\left(\mathrm{~km} \mathrm{~s}^{-1}\right)$ & $\cdots$ & -6.2 & $\cdots$ & -4.3 & $\cdots$ & 0.4 & $\mathrm{U}(0.2,0.4)$ & 82 & $\cdots$ \\
\hline Absorber-SIS & $160\left(\mathrm{~km} \mathrm{~s}^{-1}\right)$ & $\mathrm{G}(80,10)$ & -5.06 & $\cdots$ & -8.23 & $\cdots$ & $\cdots$ & $\cdots$ & $\cdots$ & $\cdots$ \\
\hline
\end{tabular}

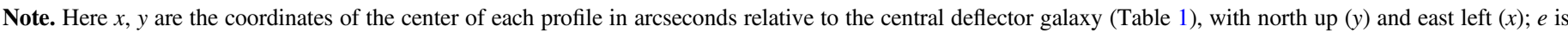

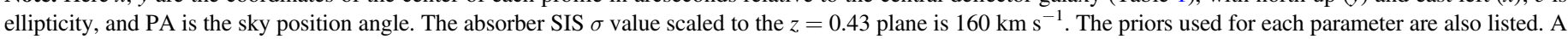

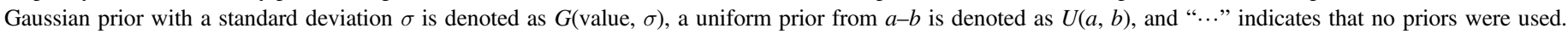

cluster $z=0.43$ lens plane for purposes of optimizing the Glafic lens model, while it is rescaled to $z=0.77$ for purposes of lens reconstruction and analysis of circumgalactic $\mathrm{Mg}$ II absorption.

The lens model is constrained by multiple images of individual star-forming regions within arc 2. The arc has a clear fold-image symmetry in its visual morphology, in which several individual regions can be identified (as also discussed by Dai et al. 2020). We use three prominent regions spanning the extent of the arc (denoted as A, B, and C; multiple images are labeled as A1, A2, A3, etc. in Figure 2). Further multiple images $(\mathrm{C} 1, \mathrm{C} 2, \mathrm{C} 3)$ are seen around the perturber galaxy located to the north of arc 2 (Figure 1), along with a counterimage, $\mathrm{C} 4$. This multiplicity of region $\mathrm{C}$ provides good lens model constraints on the perturber. Collectively, these regions pinpoint the location of the critical curve through arc 2 . We also include the counterimage of arc 2 (images A3, B3, and C5 in Figure 2) as a constraint on the lens model. This counterimage was initially identified based on consistent color and surface brightness, and we spectroscopically confirmed its nature as a multiple image of arc 2 with our 2020 data.

We fit the lens model allowing all NFW parameters to vary, with a constraint on ellipticity to prevent overfitting of the model. Priors are placed on the SIE and SIS profiles to best fit the constraints. Glafic determines the best-fit parameters using a downhill simplex method to find the region of minimal $\chi^{2}$. The values of the best-fit model are presented in Table 2 along with the adopted priors. We note that the NFW profile mass is in good agreement with expectations based on the cluster velocity dispersion (Bayliss et al. 2011). Figure 2 shows the location of the critical curve and predicted image positions for the best-fit model.

The southern giant arc (arc 1) was not used in the modeling of this system and thus offers a key test of the lens model. Our spectroscopic data and the Hubble Space Telescope (HST) imaging indicate that the arc is a single highly magnified image, which our lens model accurately reproduces. We also note that our lens model produces the same general features as the model of Dai et al. (2020), although there are some differences in the orientation of the critical curve beyond the region of the giant arcs and $\mathrm{Mg}$ II absorber galaxy, where we lack strong lensing constraints.

From the lens model, we determine the magnification factor of the $z=0.77$ galaxy to be $\mu=3.1$, calculated as the average areal magnification within a 2.5 box centered on the galaxy. We estimate the uncertainty to be approximately $10 \%$ or \pm 0.3 in $\mu$, corresponding to a spatial offset of $1^{\prime \prime}$. The magnification is reasonably precise, since the lensing potential is well constrained from the two $z \simeq 3$ arcs in this vicinity.

To calculate the impact parameters relevant for analysis of spatial CGM structure in our data, we use the lens model to raytrace the position of each KCWI spaxel to the $z=0.77$ absorber plane. The locations of the absorber galaxy and two arcs in this plane are shown in Figure 3. The center of the absorber galaxy is defined as the point of maximum continuum flux (marked in Figure 3 for both image and source plane), and the impact parameter is calculated for all spaxels as the radial distance 


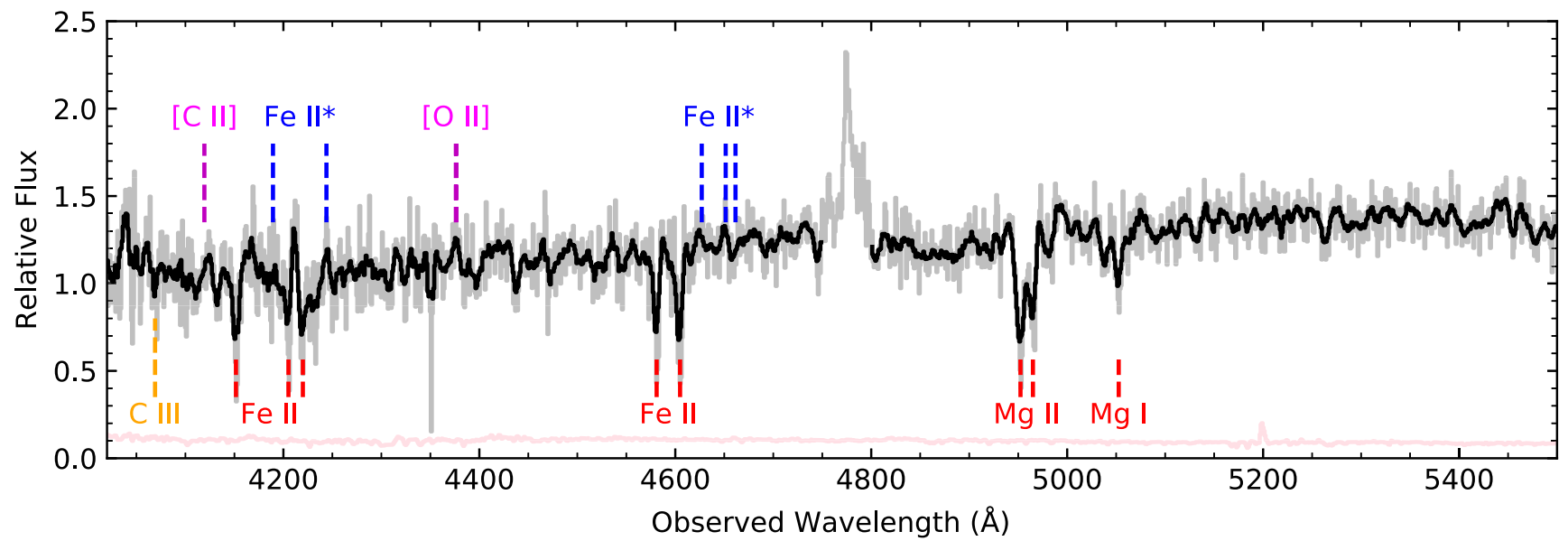

Figure 4. The KCWI spectrum of the absorber galaxy. The unsmoothed spectrum is shown in gray, while the black line is a running median over 7 pixels. The error spectrum is shown in pink. The prominent broad emission feature at $\sim 4770 \AA$ is scattered Ly $\alpha$ emission from the background $z \simeq 2.9$ arcs. Notable spectral features of the absorber galaxy are labeled (for $z=0.771$ ). Color coding indicates the physical origin of each line (yellow, stellar photospheric absorption; red, interstellar absorption; blue, fluorescent fine-structure emission; magenta, nebular emission). Interstellar absorption from $\mathrm{Mg}$ II, $\mathrm{Mg}$ I, and $\mathrm{Fe}$ II are prominent in the spectrum. Fine-structure $\mathrm{Fe}_{\mathrm{II}}^{*}$ and nebular [O II] emission are weakly detected.

from the galaxy center in the $z=0.77$ plane. We estimate the uncertainty by varying the location of the lensing critical curve and calculating the change in the impact parameter in the $z=0.77$ plane. An offset of $1^{\prime \prime}$ in the $z=2.92$ critical curve results in fractional changes of only $\lesssim 3 \%$ (one standard deviation) with $<1 \%$ change in the average values for the absorber and arc regions shown in Figure 3. We view such an offset as rather conservative, given the stringent constraints on the critical curve in this region, and thus estimate typical fractional uncertainties $\sigma \lesssim 3 \%$ in impact parameter due to the lens model.

Figure 5 shows the orientation of the absorber galaxy major axis, as determined by isophote orientation in the reconstructed HST F160W image. The lens model indicates that both arcs subtend azimuthal angles in the $z=0.77$ plane near the minor axes of the absorber galaxy, where we may expect outflow signatures (if present) to be prominent (e.g., Lan \& Mo 2018; Martin et al. 2019).

\section{Physical Properties}

\subsection{Systemic Redshift}

An accurate systemic redshift of the absorber galaxy is needed to assess the CGM kinematics relative to the host galaxy. Figure 4 shows the extracted KCWI spectrum of the absorber galaxy with prominent features labeled. The strongest lines are from interstellar absorption of $\mathrm{Mg}$ II and Fe II, which are typically blueshifted relative to the stars. The most promising systemic features covered by the spectrum are weak nebular emission lines ([O II] and [C II]) and photospheric C III $\lambda 2997$ absorption. Finestructure $\mathrm{Fe} \mathrm{II}^{*}$ fluorescent emission features can also be used to estimate the systemic redshift.

We fit Gaussian profiles to the emission features [O II] $\lambda \lambda 2471$, $\mathrm{Fe} \mathrm{II}^{*} \lambda 2396$, and $\mathrm{Fe} \mathrm{II}^{*} \lambda 2626$ in order to determine the systemic redshift. Each line is weakly detected at a signal-to-noise ratio (S/ $\mathrm{N}$ ) of 4-5 (while the remaining nebular, fluorescent, and stellar features labeled in Figure 4 are not significantly detected, with $\mathrm{S} /$ $\mathrm{N}<3$ ). We adopt the weighted mean best-fit redshift of these three lines: $z_{\text {sys }}=0.77099 \pm 0.00011$. We note that the $\lambda 2396$ and $\lambda 2626$ lines are typically the strongest of the available Fe II* transitions and are also those that most accurately trace the systemic velocity (typically within $50 \mathrm{~km} \mathrm{~s}^{-1}$; e.g., Kornei et al. 2013 ). Given that a systematic difference of $\sigma\left(z_{\mathrm{sys}}\right) \simeq 0.0002$ is possible for the fine-structure emission, and the [O II] fit has a redshift uncertainty $\sigma(z)=0.0004$, we caution that the adopted redshift uncertainty could be underestimated. Nonetheless, all three lines are consistent with the mean $z_{\text {sys }}$ within their $1 \sigma$ uncertainties and within $\Delta z \leqslant 0.00013$ (or $<25 \mathrm{~km} \mathrm{~s}^{-1}$ ) in an absolute sense.

\subsection{Mg II Absorption Line Kinematics}

Using the reduced KCWI data, we smoothed the flux measurements using a 2D Gaussian filter of FWHM $=0.15$ to increase the $\mathrm{S} / \mathrm{N}$ while preserving the spatial resolution $(\mathrm{FWHM}=1$ !" 0$)$. From there, we measured the absorption strength and kinematics of $\mathrm{Mg}$ II $\lambda \lambda 2796,2803$ at redshift $z=0.77$ in both arcs, as well as the absorber galaxy. To quantify the Mg II absorption properties, we selected all spaxels corresponding to the absorber galaxy and the two background arcs with a minimum continuum $\mathrm{S} / \mathrm{N}>5 \sigma$ per spectral pixel, giving us a combined total of 280 selected spaxels.

At each spaxel position, the spectrum near $4950 \AA$ was fit using a sum of three Gaussian profiles corresponding to the Mg II doublet at $z=0.77$ and the Si II $\lambda 1260$ absorption line at $z=2.92$ from the background arcs. The triple Gaussian fit is parameterized by the rest-frame equivalent width $\left(W_{0}\right)$, velocity offset $(v)$, and velocity dispersion $(\sigma)$ for the three absorption lines, allowing us to characterize the spatial trends of these parameters. Figure 5 provides examples of the spectral fits for various spaxels within the absorber galaxy and both gravitational arcs. It is important to note that the models adopted a lower limit to the velocity dispersion given by the spectral resolution of KCWI (FWHM $=2.4 \AA$ ). The best-fit line widths were then corrected for this instrumental resolution to give us the intrinsic velocity dispersion measurements for each spaxel. Our fits used a common redshift and velocity dispersion for both $\mathrm{Mg}$ II lines in each spaxel, providing more robust fits and minimizing spurious fits to noise in low-S/N regions. Spatial maps of the best-fit equivalent widths, velocity offsets, and dispersions of $\mathrm{Mg}$ II absorption are shown in Figure 6. In addition to spatially resolved spectra, we measured spatially integrated spectra of the absorber galaxy and background arcs 


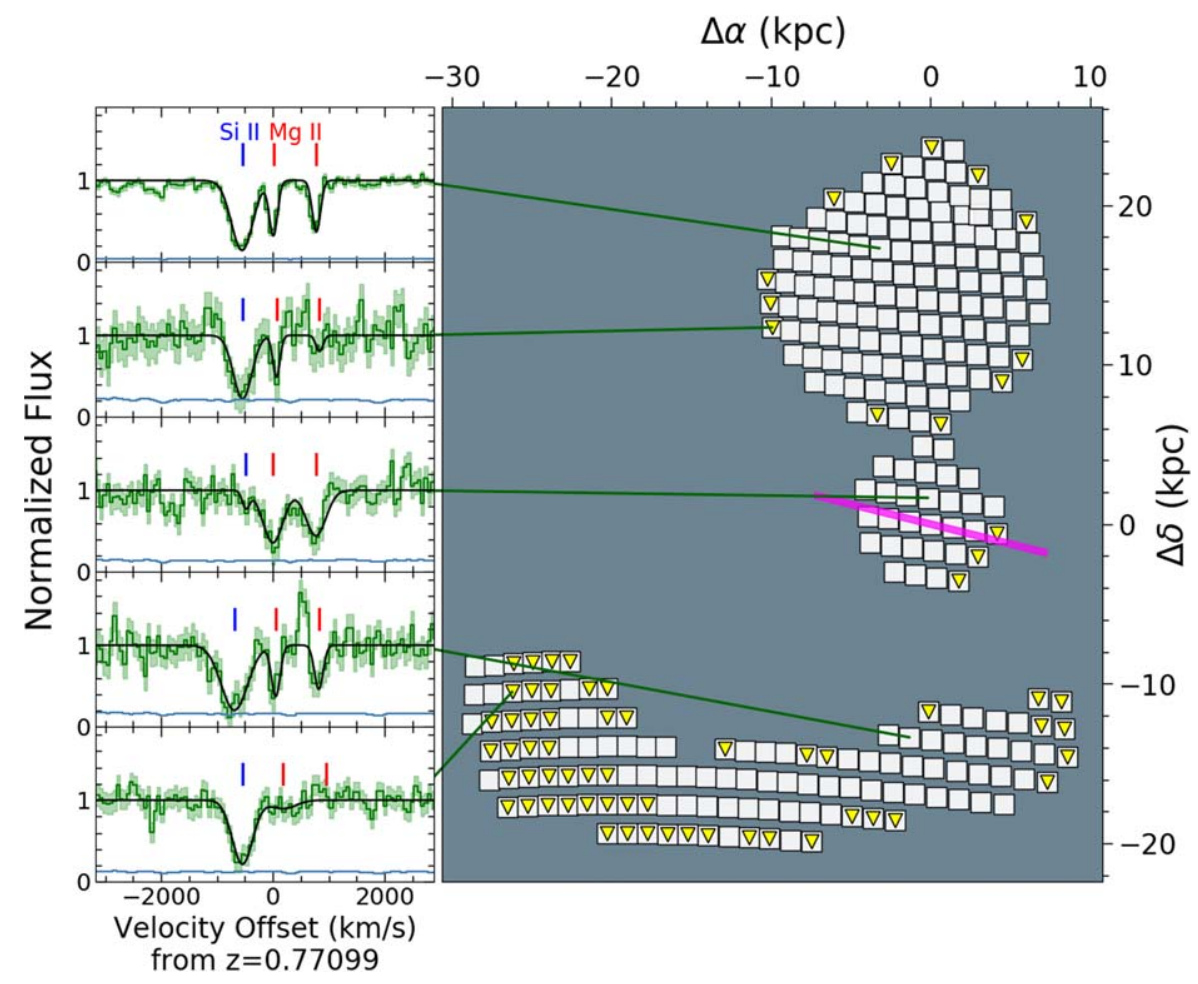

Figure 5. Spectra of individual spaxels showing absorption profiles from $\mathrm{Mg}$ II seen toward the absorber galaxy and background arcs (Section 4). The image on the right shows spaxel positions in the $z=0.77$ plane. Each spaxel shown has a continuum $\mathrm{S} / \mathrm{N}>5 \sigma$ per spectral pixel near the Mg II absorption, while yellow triangles denote spaxels where $\mathrm{Mg}$ II absorption is not detected $(<5 \sigma)$. The displayed spectra span a range of $\mathrm{S} / \mathrm{N}$ in both the continuum and $\mathrm{Mg}$ II absorption, including Mg II detections and nondetections, illustrating the data quality. Raw spectra in each panel (green) are overlaid with a triple Gaussian fit (black) corresponding to the three most prominent absorption features: Si II $\lambda 1260$ in the background arcs at $z=2.92$ and the Mg II $\lambda \lambda 2796,2803$ doublet at $z=0.77$, which is the main subject of this work. The error spectrum in each spaxel is shown in blue. The pink line denotes the morphological major axis of the absorber galaxy in the $z=0.77$ plane; this axis has an orientation angle of $\theta \approx 76^{\circ}$.
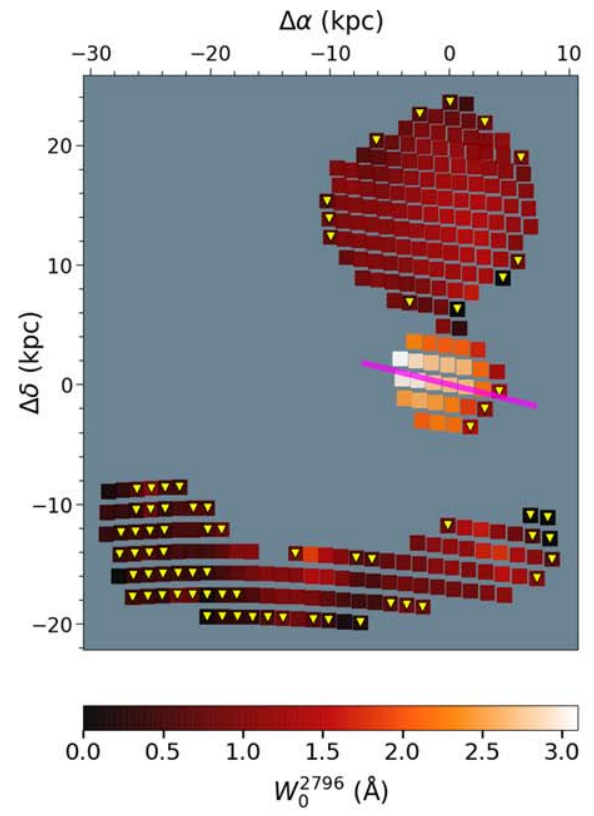
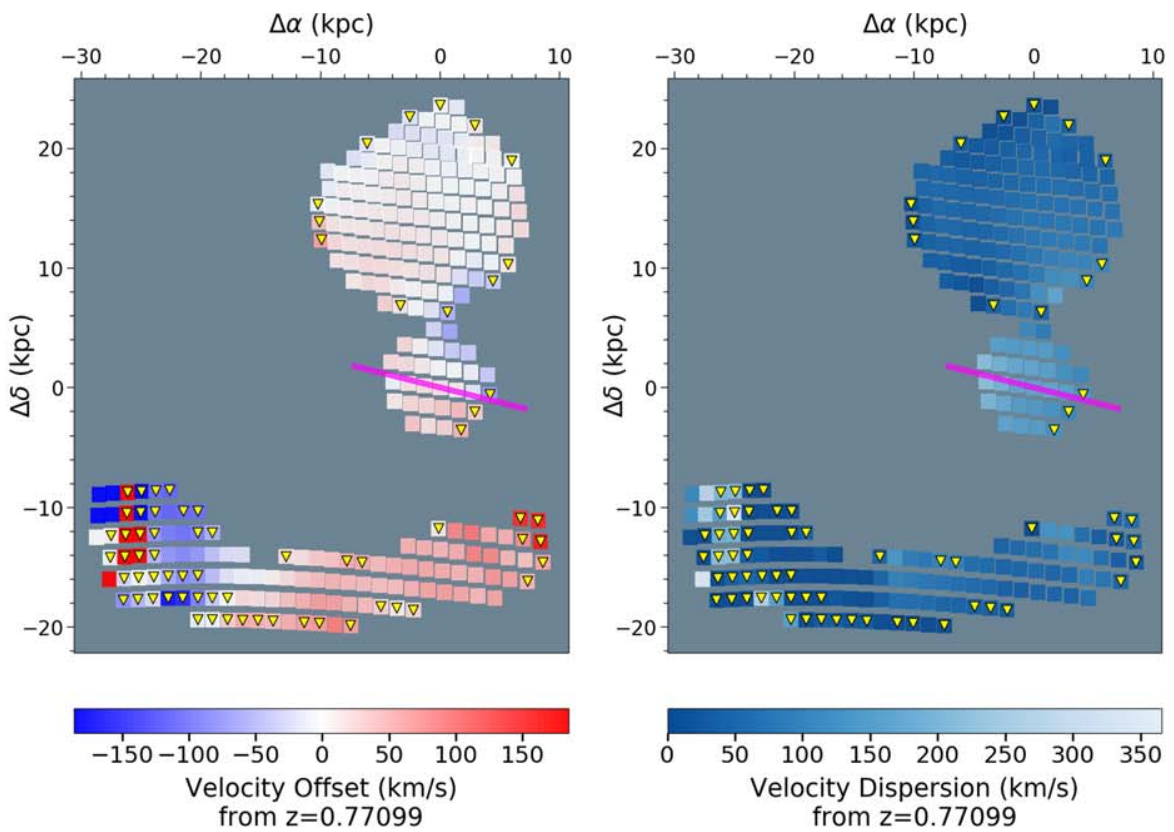

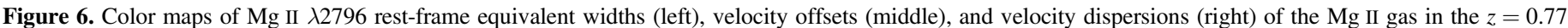

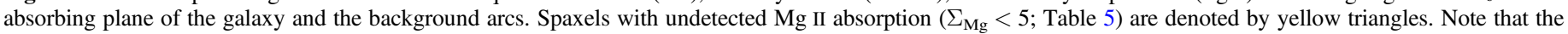
spaxels in the color maps are distinguished by their offset separation $(\Delta \alpha, \Delta \delta)$ with respect to the center of the absorber galaxy (see Figure 3).

to compare the fits at high-S/N (see Section 5.2). The fitted parameters of the integrated spectra for the galaxy-arc system are provided in Table 4.
Of the spaxels that have continuum $\mathrm{S} / \mathrm{N}>5 \sigma$, we check the detection of $\mathrm{Mg}$ II absorption within the fit profiles using a $\chi^{2}$ test. We compare how the addition of Mg II components in our fit 


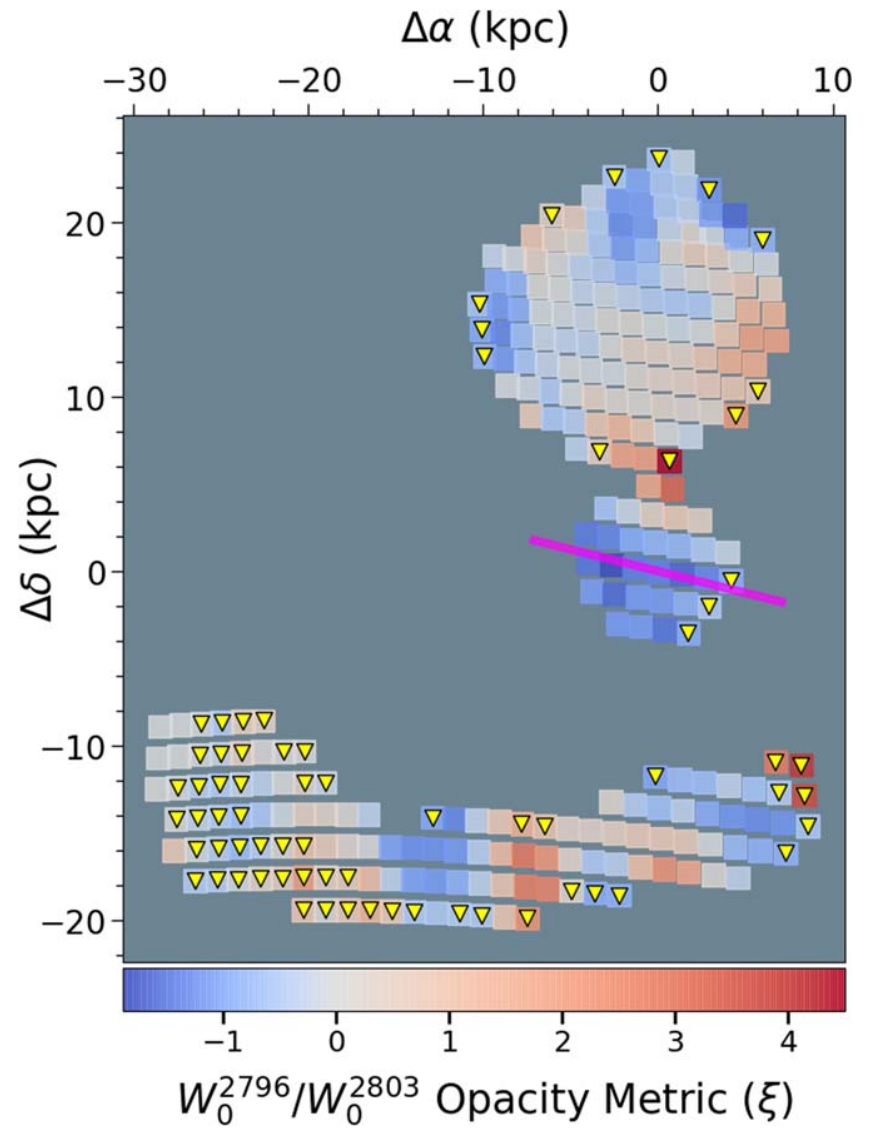

Figure 7. Color map of the opacity metric $(\xi)$ of $W_{0}^{2796} / W_{0}^{2803}$ in the absorber plane in units of standard deviations. Values of $\sim 0$ correspond to optically thick gas, whereas values $\lesssim-1$ and $\gtrsim 1$ may indicate optically thin gas and nonphysical absorption ratios, respectively.

improves the $\chi^{2}$ value compared to a fit with only the Si II absorption and thereby determine the detection significance $\Sigma_{\mathrm{Mg}}$ of $\mathrm{Mg}$ II absorption in units of standard deviations. Spaxels whose $\mathrm{Mg}$ II absorption detection significance is marginal $(<5 \sigma)$ are considered nondetections and will be denoted in plots as either yellow triangles (e.g., Figures 6 and 7) or open data points (e.g., Figure 9). A full list of the fitted parameters, $\mathrm{Mg}$ II significance values $\left(\Sigma_{\mathrm{Mg}}\right)$, and continuum $\mathrm{S} / \mathrm{N}$ for each spaxel is in Table 5. The table also contains the offset separation $(\Delta \alpha, \Delta \delta)$ of each spaxel with respect to the galaxy in the $z=0.77$ absorber plane $(\mathrm{kpc})$, as well as the impact parameter of each spaxel in the absorber plane $(D)$.

The color maps show strong $\mathrm{Mg}$ II absorption with $W_{0}^{2796}>1$ $\AA$ across a large area in both arcs, indicating the widespread presence of cool, metal-enriched circumgalactic gas. In addition, the absorption strength in the arcs is not uniform, indicating a clumpy medium similar to the findings of Lopez et al. (2018), who also performed a similar tomographic observation of a $z=0.98$ galaxy system (with a somewhat lower inferred halo mass of $M_{\text {halo }} \sim 10^{11} M_{\odot}$; see Section 4.4) at impact parameters of $\approx 15-90 \mathrm{kpc}$. The Mg II absorption fits from the larger impact parameters of arc 1 suggest inhomogeneities within the CGM. It is likely that the CGM can span the entirety of arc 1; however, the distribution of $\mathrm{Mg}$ II that is well detected is relatively close (within $\sim 40 \mathrm{kpc}$ ) to the absorber galaxy. While strong Mg II absorption is prominently seen from the CGM, the gas in the arcs shows little bulk motion relative to the galaxy; at first glance, the velocity
Table 3

Photometry of the $z=0.77 \mathrm{Mg}$ II Absorber Galaxy

\begin{tabular}{lc}
\hline \hline Filter & AB Magnitude \\
\hline HST/ACS F606W & $22.47 \pm 0.02$ \\
HST/ACS F814W & $21.45 \pm 0.02$ \\
HST/WFC3-IR F110W & $20.91 \pm 0.02$ \\
HST/WFC3-IR F160W & $20.56 \pm 0.02$ \\
Pan-STARRS $g$ & $23.07 \pm 0.24$ \\
Pan-STARRS $r$ & $22.45 \pm 0.22$ \\
Pan-STARRS $i$ & $21.47 \pm 0.09$ \\
Pan-STARRS $z$ & $21.65 \pm 0.14$ \\
\hline
\end{tabular}

Note. The photometry is used to determine the stellar mass and SFR.

offsets relative to the central galaxy are fairly small $\left(|v| \lesssim 80 \mathrm{~km} \mathrm{~s}^{-1}\right)$, and the typical velocity dispersions seen in the background arcs are only $\simeq 50 \mathrm{~km} \mathrm{~s}^{-1}$. Since we do not detect any stellar or nebular features to determine the systematic redshift of the galaxy, we caution that velocity offsets are converted with respect to the average $\mathrm{Mg} \mathrm{II} \mathrm{absorption} \mathrm{redshift} \mathrm{of} \mathrm{the} \mathrm{galaxy-arc}$ system $(z \approx 0.7711)$. We anticipate that the true systemic redshift is likely underestimated and could differ by up to $\sim 50 \mathrm{~km} \mathrm{~s}^{-1}$, based on the differences in velocity offsets between the two arcs. Relative velocities within the CGM are nonetheless unaffected. These "kinematically cold" arcs contrast with the larger velocity dispersion $\left(\sigma \approx 170 \mathrm{~km} \mathrm{~s}^{-1}\right)$ and blueshift observed toward the Mg II absorber galaxy itself ("down-the-barrel" kinematics; see Table 4), which is presumably due to outflowing gas driven by star formation in the galaxy.

\subsection{Stellar Mass}

We derive the stellar mass and other stellar population properties from the integrated spectral energy distribution. We measure broadband fluxes in several filters from observations with the HST (programs GO-12368 and GO-15378) and PanSTARRS (Chambers et al. 2016), summarized in Table 3. To calculate magnitudes, we sum the flux within a 2 !" $25 \times 3$ "! 25 aperture that captures the full spatial extent detected in HST imaging. This aperture does not capture extended flux in PanSTARRS images due to seeing, so we subtract 0.27 from the Pan-STARRS magnitudes to match the HST photometry. We adopt a minimum systematic uncertainty of $2 \%$ in photometric fluxes, although the true uncertainty is likely even higher (Ilbert et al. 2006). Photometric measurements are then fit with the stellar population synthesis code FAST (Kriek et al. 2009). We adopt Bruzual \& Charlot (2003) spectral templates with a Chabrier initial mass function, solar metallicity, a Calzetti et al. (2000) dust attenuation curve, and an exponentially declining star formation history. The resulting best-fit stellar mass is $\log \left(M_{*} / M_{\odot}\right)=9.6_{-0.1}^{+0.2}$, corrected for a lensing magnification factor $\mu=3.1 \pm 0.3$. Adopting a constant star formation history, the best fit is $\log \left(M_{*} / M_{\odot}\right)=9.8 \pm 0.1$ and $\mathrm{SFR}=10 \pm 5 M_{\odot} \mathrm{yr}^{-1}$. The specific SFR is consistent with the "main sequence" of star-forming galaxies at $z \approx 0.8$ $\left(\approx 10^{-9.0} \mathrm{yr}^{-1}\right.$; e.g., Whitaker et al. 2014).

\subsection{Halo Mass and Circular Velocity}

The dark matter halo properties, in particular the expected rotation curve, are important for interpreting measurements of the circumgalactic gas kinematics. We estimate the dark matter halo mass using the stellar-to-halo mass relation of Behroozi 
et al. (2013). For the source (absorber galaxy) redshift and stellar mass, the expected halo virial mass and radius are $\log \left(M_{\text {halo }} / M_{\odot}\right)=11.6 \pm 0.2$ and $R_{\text {vir }}=115 \pm 20 \mathrm{kpc}$ (defined as $R_{\text {vir }}=\left(3 M_{\text {halo }} / 4 \pi \times 200 \rho_{c}\right)^{1 / 3}$, with $\rho_{c}$ being the critical density at $z=0.77$ ), accounting for both the uncertainty in the stellar mass and the $\sim 0.1$ dex scatter in the stellar-to-halo mass relation. The expected circular rotation velocity $v_{c}$ is relatively insensitive to halo mass (e.g., Bullock \& Boylan-Kolchin 2017). Over the range of radii of interest here-from $\sim 10 \mathrm{kpc}$ to of order half the virial radius-a halo mass $\log \left(M_{\text {halo }} / M_{\odot}\right)=11.6 \pm 0.2$ corresponds to $v_{c} \approx 100 \pm$ $20 \mathrm{~km} \mathrm{~s}^{-1}$. For a purely dispersion-supported halo, the expected velocity dispersion is $\sigma_{m}=\frac{v_{c}}{\sqrt{2}}=70 \pm 10 \mathrm{~km} \mathrm{~s}^{-1}$, assuming an isothermal profile.

\section{Spatial and Kinematic Structure of the CGM}

\subsection{Optical Depth and Covering Fraction of $\mathrm{Mg}$ II}

In this section, we examine whether variation in the $\mathrm{Mg}$ II equivalent width is caused by differences in the gas covering fraction, column density, or a combination. A key diagnostic is the optical depth of $\mathrm{Mg}$ II absorption revealed by the doublet ratio. If a lower equivalent width is due to a low gas column density, then we expect to see a larger ratio of $W_{0}^{2796} / W_{0}^{2803}>1$ in optically thin regions of the arcs, whereas if it is due to a lower covering fraction, we expect a ratio closer to 1 in the regions of low equivalent width.

Figure 7 illustrates the equivalent-width line ratios of $\mathrm{Mg}$ II $\lambda 2796\left(W_{0}^{2796}\right)$ and $\mathrm{Mg}$ II $\lambda 2803\left(W_{0}^{2803}\right)$. The ratios are displayed in terms of an "opacity metric" $\xi$, where

$$
\xi=\frac{1-\frac{W_{0}^{2796}}{W_{0}^{2803}}}{\mathrm{SD}\left(\frac{\mathrm{W}_{0}^{2796}}{\mathrm{~W}_{0}^{2803}}\right)},
$$

where $\operatorname{SD}(x)$ is the standard deviation of the line ratio $x$ obtained by propagating the uncertainties. Optically thick gas is characterized by $W_{0}^{2796} \simeq W_{0}^{2803}$, so our metric classifies optically thick gas at $\xi=0$. Optically thin gas corresponds to values $\xi<0$; for example, a value of $\xi=-3$ would indicate that the $\mathrm{Mg}$ II absorption is not optically thick at $3 \sigma$ significance. Values $\xi>0$ are nonphysical for pure absorption, although in principle, such values can arise from saturated absorption combined with emission line filling.

The results show that the two arcs mostly vary within $\pm 1 \sigma$ of $\xi=0$, which is indicative of optically thick gas. We see minimal indication of optically thin gas, and nonphysical values are located in spaxels near the edges of the arcs where the absorption signal is not as strong, possibly resulting from spurious fits. The low variation among the line ratios indicates that the low equivalent widths are driven primarily by kinematics and spatial covering fraction, rather than column density. Therefore, the variation in equivalent width appears to indicate a patchy spatial distribution of $\mathrm{Mg}$ II gas, similar to the results from Lopez et al. (2018, 2020).

\subsection{Equivalent Width versus Impact Parameter}

To achieve a better understanding of the spatial distribution of $\mathrm{Mg}$ II gas in Figure 6, we compared the absorption profiles between the absorber galaxy and the two gravitational arcs through both a stacked spaxel analysis (Figure 8 and Table 4) and as a function of impact parameter $D$ (Figure 9). We caution that individual spaxels are not independent due to the seeing, causing the correlated patterns seen in arc 2 measurements. The true spatial resolution element corresponds to $\simeq 10$ spaxels $\left(\simeq 15 \mathrm{kpc}^{2}\right)$ and spans $\sim 2-8 \mathrm{kpc}$ in Figure 9. To account for the true resolution, we provided local regression curves to both arcs in Figure 9 that were obtained through locally weighted scatterplot smoothing (LOWESS) methods. These LOWESS curves show the overall trends of the $\mathrm{Mg}$ II gas in the arc sight lines while smoothing over the correlated spaxel patterns caused by the seeing.

The results show a moderate $W_{0}-D$ anticorrelation in both arcs, with absorption falling below the detection threshold for individual spaxels at impact parameters $D \gtrsim 25 \mathrm{kpc}$ from the center of the galaxy (see Figure 8), limiting our analysis of the $\mathrm{Mg}$ II distribution to within $\sim 10-25 \mathrm{kpc}$. The LOWESS curves indicate that the average equivalent width of $\mathrm{Mg}$ II absorption seen in arc 1 is similar to that in arc 2 at a fixed impact parameter, and both arcs exhibit lower equivalent widths than the absorber galaxy. If there was uniform homogeneity in the gas, we would expect similar measurements of $\mathrm{Mg}$ II gas on both sides of the CGM (probed by the two arcs). However, there are variations of equivalent width within arc 1 (about $2 \sigma-3 \sigma$ at some impact parameters) that are not apparent in arc 2 at similar impact parameters, resulting in an inconsistency with a purely symmetric distribution of circumgalactic gas at a more detailed level than what occurs on average. This anisotropy is broadly consistent with $\mathrm{Mg}$ II distributions seen around other galaxies (e.g., Lopez et al. 2018, 2020) and is perhaps unsurprising given that $\mathrm{Mg} \mathrm{II}$ is observed to vary strongly on kpc scales from lensed quasar sight lines (e.g., Ellison et al. 2004) and as a function of azimuthal angle in composite samples (e.g., Bordoloi et al. 2011).

We compare our arc data in Figure 9 with quasar sight lines from 182 intermediate-redshift galaxies $(0.072 \leqslant z \leqslant 1.120)$ in the Mg II Absorber-Galaxy Catalog (Nielsen et al. 2013a), as well as tomographic measurements of two intermediate-redshift galaxy systems at $z=0.98$ and 0.73 (with $\log \left(M_{\text {halo }} / M_{\odot}\right) \approx 11.0$ and 11.6, respectively) described in Lopez et al. (2018, 2020). In general, our data are in agreement with the trend of the quasar statistics $\left(\log W_{r}(2796)=\alpha_{1} D+\alpha_{2}\right.$, where $\alpha_{1}=-0.015 \pm$ 0.002 and $\alpha_{2}=0.27 \pm 0.11$; Nielsen et al. 2013a) and fall well within the spread of the quasar sight lines (RMSE $\approx 0.66$ ). This result is consistent with other individual galaxy measurements from lensed arc tomography (Lopez et al. 2018, 2020).

A striking feature of Figure 9 is how closely the arc tomography data track the average of QSO sight lines. Our measurements from the CSWA 38 system, as well as the two systems studied by Lopez et al. $(2018,2020)$, show much smaller scatter than the quasar samples. We consider two possible explanations for the difference in scatter. On one hand, scatter may arise from halo-to-halo variations in the CGM of different galaxies as traced by $\mathrm{Mg}$ II absorption. The extent and equivalent width of $\mathrm{Mg}$ II is correlated with global galaxy properties such as stellar mass, SFR, environment, and redshift (e.g., Bordoloi et al. 2011; Nielsen et al. 2013a). The three galaxies studied with arc tomography are similar in terms of global properties, which may explain their relative consistency in $\mathrm{Mg}$ II absorption equivalent width. The QSO comparison sample in Figure 9 includes a broader range of galaxy properties, which can explain at least some of the larger scatter. Another effect is that small-scale fluctuations within the CGM around individual galaxies can give rise to larger scatter toward the QSO sight lines. Indeed, lensed QSO systems reveal 

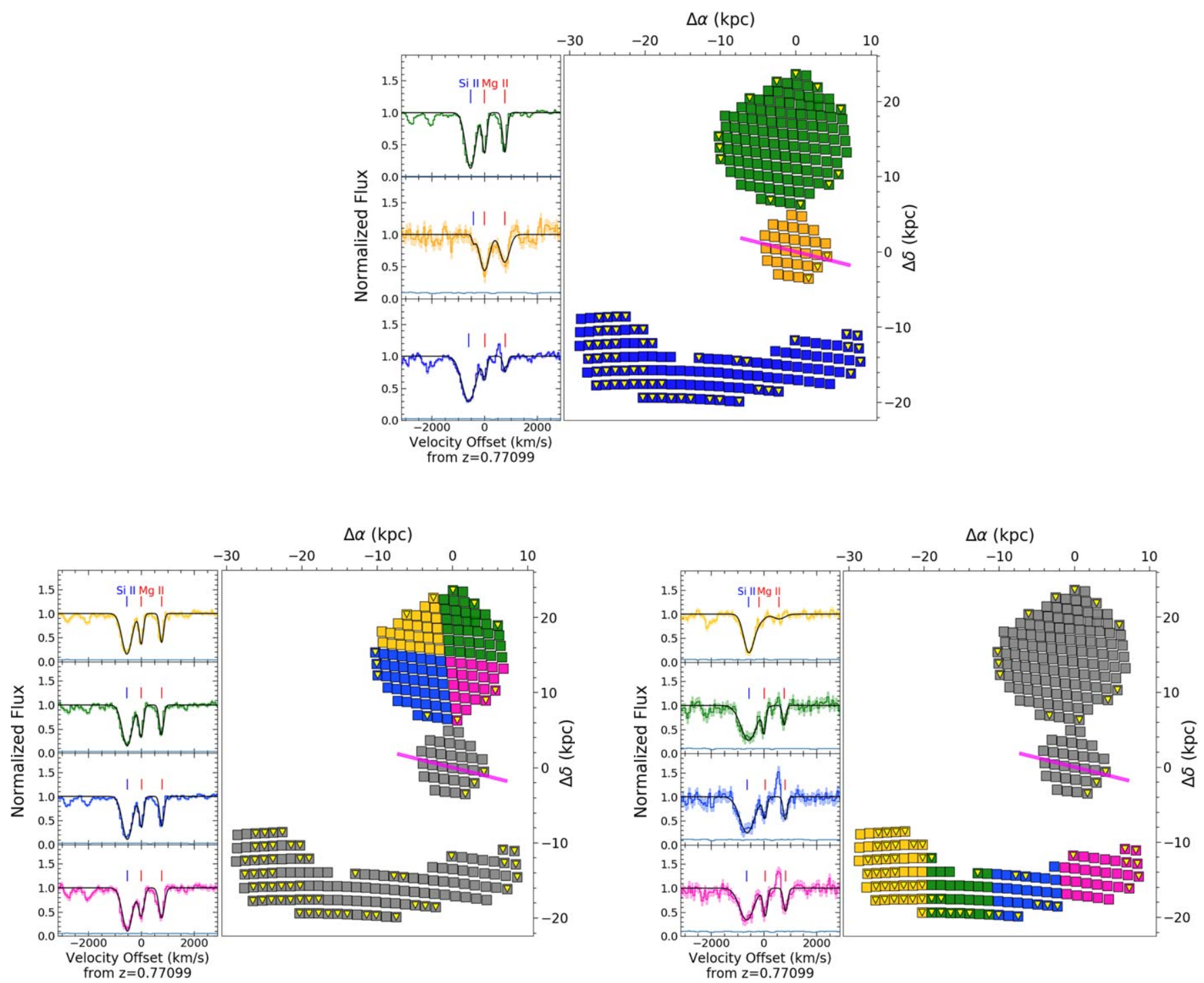

Figure 8. Top: spatially integrated spectra of the absorber galaxy (orange) and background arcs (blue and green), showing the high-S/N absorption signal obtained from summing all spaxels within each object. The background arcs show prominent Si II $\lambda 1260$ absorption at the arc redshifts $(z \simeq 2.92)$, unrelated to the Mg II absorption associated with the $z=0.77$ galaxy. The spatial regions corresponding to these integrated spectra are shown at right in the $z=0.77$ plane. Bottom: similar spectra from binned spaxels for different regions of arcs 1 (left) and 2 (right), each separated into four regions of comparable area. The best-fit measurements for the Mg II $\lambda 2796$ rest-frame $W_{0}, v$, and $\sigma$ for these spatially binned regions, as well as the average impact parameter $D$, are reported in Table 4.

Table 4

Mg II $\lambda 2796$ Absorption Distribution and Kinematics (Figure 8)

\begin{tabular}{|c|c|c|c|c|}
\hline & $\begin{array}{c}D \\
(\mathrm{kpc})\end{array}$ & $\begin{array}{l}W_{0} \\
(\AA)\end{array}$ & $\begin{array}{c}v \\
\left(\mathrm{~km} \mathrm{~s}^{-1}\right)\end{array}$ & $\begin{array}{c}\sigma \\
\left(\mathrm{km} \mathrm{s}^{-1}\right)\end{array}$ \\
\hline Galaxy (orange) & $1.79 \pm 0.97$ & $2.35 \pm 0.20$ & $4.08 \pm 16.44$ & $167.30 \pm 14.84$ \\
\hline Region 1 (yellow) & $29.35 \pm 2.68$ & $0.69 \pm 0.30$ & $-155.28 \pm 86.51$ & $199.33 \pm 50.51$ \\
\hline Region 2 (green) & $23.61 \pm 2.34$ & $0.75 \pm 0.15$ & $9.54 \pm 10.04$ & $24.92 \pm 28.11$ \\
\hline Region 3 (blue) & $18.07 \pm 2.22$ & $0.73 \pm 0.14$ & $43.55 \pm 11.68$ & $43.41 \pm 20.44$ \\
\hline Arc 2 (green) & $12.91 \pm 4.43$ & $1.21 \pm 0.09$ & $5.63 \pm 5.27$ & $56.73 \pm 7.83$ \\
\hline Region 1 (yellow) & $19.13 \pm 1.76$ & $1.11 \pm 0.09$ & $3.81 \pm 5.44$ & $44.26 \pm 9.38$ \\
\hline Region 2 (green) & $19.01 \pm 2.36$ & $1.26 \pm 0.09$ & $-1.62 \pm 4.98$ & $53.73 \pm 7.63$ \\
\hline Region 3 (blue) & $12.70 \pm 3.06$ & $1.16 \pm 0.09$ & $12.57 \pm 5.31$ & $49.22 \pm 8.56$ \\
\hline Region 4 (pink) & $11.54 \pm 2.28$ & $1.40 \pm 0.12$ & $3.22 \pm 7.42$ & $83.70 \pm 9.23$ \\
\hline
\end{tabular}




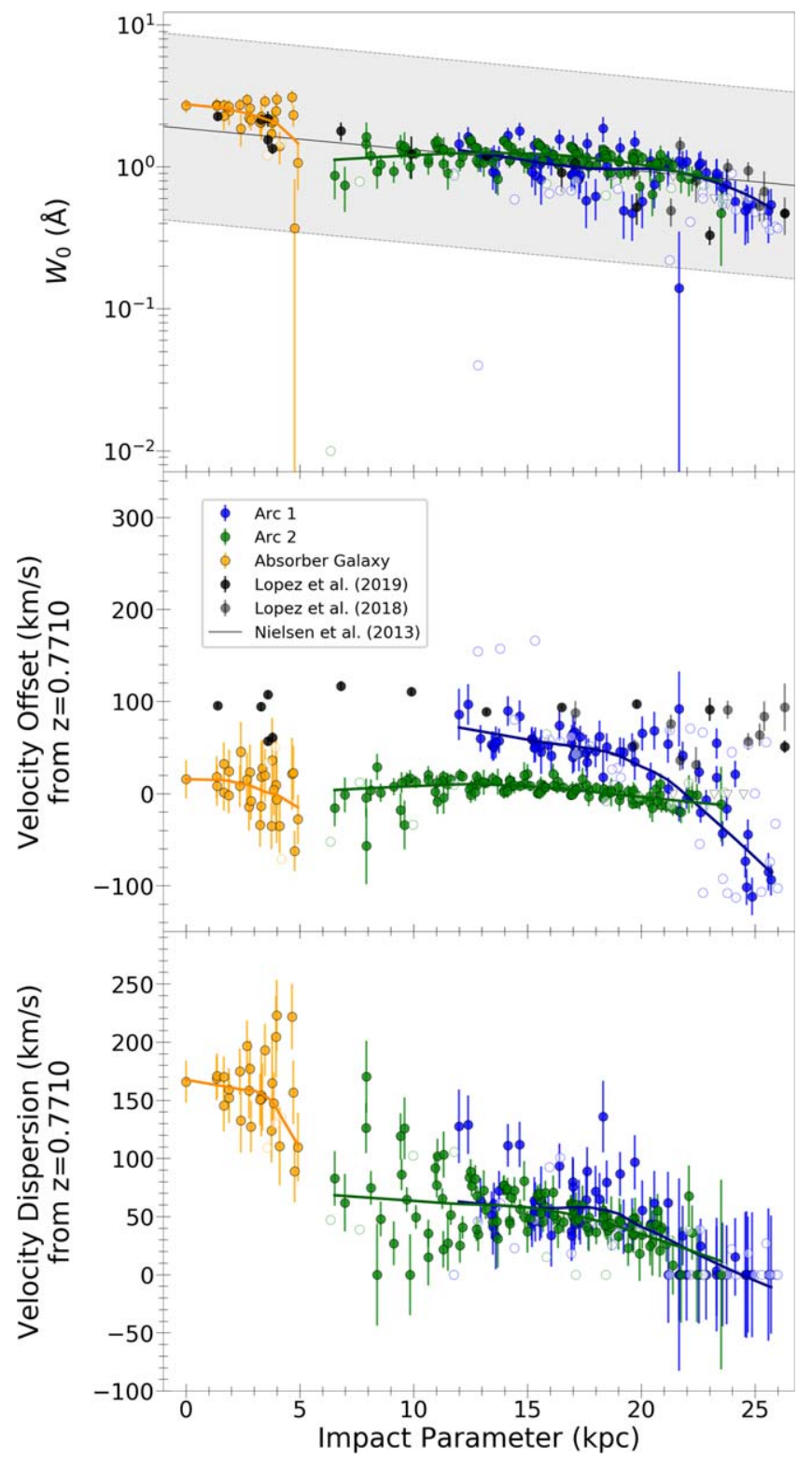

Figure 9. The Mg II $\lambda 2796$ rest-frame equivalent width (top), velocity offset (middle), and velocity dispersion (bottom) as a function of impact parameter $D$ in the absorber plane for arc 1 (blue), arc 2 (green), and the absorber galaxy (orange). The spaxels in each arc with undetected $\mathrm{Mg}$ II absorption $\left(\Sigma_{\mathrm{Mg}}<5\right)$ are denoted by open data points of the equivalent colors in each plot. Error bars denote $1 \sigma$ uncertainty in the best-fit parameters of each spaxel. The curves in each arc were obtained through LOWESS methods to understand the trends of the $\mathrm{Mg}$ II gas in the arcs while ignoring the correlated spaxel patterns affected by the seeing. For comparison, the black and gray data points are similar lensing tomographic measurements from Lopez et al. $(2018,2020)$. In the top panel, the black line and shaded region show the maximum-likelihood fit from Nielsen et al. (2013a; for a sample of quasar-galaxy pairs) and the associated sample rms variation.

considerable variation in $\mathrm{Mg}$ II absorption on $\sim \mathrm{kpc}$ scales (e.g., Ellison et al. 2004; Chen et al. 2014; Rubin et al. 2018c). The arc tomography data have a spatial resolution of order $\sim 15 \mathrm{kpc}^{2}$, orders of magnitude larger than the effective area of the QSO sight lines. The larger cross-sectional area should average over such small-scale fluctuations and result in lower scatter for the arc tomography. We would expect similarly decreased scatter for unlensed background galaxy sight lines, which likewise subtend a much larger cross section than QSOs.
There is ample evidence to suggest that the two effects discussed above-bulk halo-to-halo variations of the CGM and intrahalo fluctuations on scales smaller than the effective resolution elements - are both relevant. Given the small available lens tomography sample, we do not attempt to distinguish their relative contributions here. However, we note that the intrahalo effect offers an avenue for probing the coherence length scale of absorption within the CGM. Scatter in $W_{r}(2796)$ versus $D$ should anticorrelate with the cross-sectional size of the background source, with a dependence on the physical size of individual CGM absorption clouds. Characterizing this scatter for sources of different sizes (e.g., QSOs versus galaxies in Figure 9) can therefore provide new information on the spatial structure of the CGM around distant galaxies.

\subsection{Angular Momentum versus Velocity Dispersion Support of CGM Gas}

Comparison of the arcs with the absorber galaxy (Figure 8 and Table 4) reveals obvious differences in gas kinematics. The absorber galaxy notably exhibits a best-fit $\mathrm{Mg}$ II absorption velocity dispersion of $\sigma \simeq 167 \mathrm{~km} \mathrm{~s}^{-1}$, which is roughly $3.5 \times$ larger than in both $\operatorname{arcs} 1$ and $2\left(\sigma \simeq 50 \mathrm{~km} \mathrm{~s}^{-1}\right)$. This velocity dispersion largely drives the higher absorption equivalent widths seen toward the absorber galaxy. Moreover, the Mg II profile in the absorber galaxy is clearly skewed toward negative velocities, reaching $\simeq-500 \mathrm{~km} \mathrm{~s}^{-1}$, as we discuss further in Section 6. Such a broad velocity range suggests a significant outflow component seen "down the barrel" of the absorber galaxy, yet we see only modest evidence of such broad outflow velocities in the background arcs even at small impact parameters $(\sim 10 \mathrm{kpc})$. We note that while down-the-barrel measurements probe line-ofsight kinematics, the absorption can be dominated by dense gas close to (or within) the galaxy, and the velocity measurements do not tell us how far the outflowing gas extends from the galaxy. We now examine the extent to which velocity gradients in the background arcs can be attributed to bulk rotational motion of the CGM.

To further probe the velocity structure seen in the arcs, Figure 9 displays the velocity offsets and dispersions as functions of impact parameter. Our results indicate that the Mg II velocities show a modest variation within both arcs. The total range of velocity offsets spans $-110 \mathrm{~km} \mathrm{~s}^{-1} \lesssim v \lesssim 100 \mathrm{~km} \mathrm{~s}^{-1}$ and averages $v \approx 15 \pm 15 \mathrm{~km} \mathrm{~s}^{-1}$. However, most spaxels at the low end of this velocity range are at impact parameters $\sim 25 \mathrm{kpc}$, near the boundary of where we confidently detect $\mathrm{Mg}$ II absorption. The velocity gradient in arc 2 seen in Figure 9 is likely affected by spurious $\mathrm{Mg}$ II fits, as we do not see clear evidence of such a gradient in the spatially binned spectra (Figure 8). Considering only robust detections at impact parameters $<20 \mathrm{kpc}$, the total range of velocity offsets is only $\sim 0-100 \mathrm{~km} \mathrm{~s}^{-1}$. The range of best-fit velocity dispersions in the arcs is relatively large $(0 \mathrm{~km}$ $\mathrm{s}^{-1} \lesssim \sigma \lesssim 170 \mathrm{~km} \mathrm{~s}^{-1}$ ), although the majority of spaxels are near the average of $\sigma \approx 50 \pm 25 \mathrm{~km} \mathrm{~s}^{-1}$. Therefore, the typical velocity FWHM $\left(\sim 120 \mathrm{~km} \mathrm{~s}^{-1}\right)$ in any given spaxel is comparable to or larger than the variation in bulk motion seen across the entire system. The mean $\sigma$ value is similar to the expected dark matter halo velocity dispersion of $\sigma_{m}=70 \pm 10 \mathrm{~km} \mathrm{~s}^{-1}$ for a dispersionsupported system (Section 4.4; Elahi et al. 2018).

Another important feature of the $\mathrm{Mg}$ II kinematics is that there is a noticeable difference in the velocity offsets of the arcs (Figure 9), which may provide evidence of angular momentum or a biconical outflow in the system. For an approximately isothermal density 
profile, we expect that the rotation $v_{r}$ and velocity dispersion $\sigma$ are related via $v_{r}^{2} \approx v_{c}^{2}-2 \sigma^{2}$ (e.g., Burkert et al. 2010). The bulk rotation velocity is expected to be lower than the circular velocity of the potential because the radial (turbulent) pressure gradient counteracts the centripetal acceleration (see also Wellons et al. 2020). Here $v_{c}=100 \pm 20 \mathrm{~km} \mathrm{~s}^{-1}$ is the circular velocity based on the estimated halo mass (Section 4.4), and we measure an average $\sigma=50 \mathrm{~km} \mathrm{~s}^{-1}$ from the background arc sight lines. We therefore expect the bulk rotation velocity to be $v_{r}=70 \pm 30 \mathrm{~km} \mathrm{~s}^{-1}$, with a ratio $v_{r} / \sigma=1.4 \pm 0.6$. Our measured velocities imply a smaller $v_{r}$ than this but are compatible with this simple picture given the possible effects of inclination and orientation. Since the lens model suggests that the arcs do not sample the kinematic major axis (Section 3), the data do not robustly constrain the degree of rotational motion or the inclination or orientation of such possible rotation. A rotation curve measurement for the absorber galaxy, which we do not have at present, would be valuable for further constraining the angular momentum.

The galaxies in Lopez et al. $(2018,2020)$ exhibit higher velocity offsets compared to our data (Figure 9), despite the lower (but comparable) stellar masses of the host galaxies. This may further indicate that, in a relative sense, angular momentum is less important in the CGM of the CSWA 38 system studied here than in the systems studied by Lopez et al. In summary, our measurements of spatially resolved kinematics appear to be dominated by a relatively uniform velocity dispersion component, although we cannot rule out a substantial degree of rotation.

\section{Physical Interpretation of the Circumgalactic Absorption}

We now discuss the physical interpretation of velocity dispersions measured from single-component fits, which indicate typical $\sigma \approx 50 \mathrm{~km} \mathrm{~s}^{-1}$ or $\mathrm{FWHM} \approx 120 \mathrm{~km} \mathrm{~s}^{-1}$ throughout most regions of the background arcs. Highresolution spectroscopy of quasar sight lines reveals that lowionization CGM absorption occurs in discrete clouds that individually have small Doppler $b$ parameters. Such clouds would be unresolved and blended at the spectral resolution of our KCWI data (e.g., Zabl et al. 2020). Moreover, such clouds are likely far smaller than the effective $\sim 15 \mathrm{kpc}^{2}$ spatial resolution element of our data. Indeed, lensed quasar sight lines show that $\mathrm{Mg}$ II absorption profiles vary strongly on $\sim \mathrm{kpc}$ scales (e.g., Ellison et al. 2004; Chen et al. 2014). Therefore, the absorption profiles measured for individual spaxels likely represent contributions from numerous discrete clouds, each of which is spectrally unresolved and has a nonunity covering fraction even within a single resolution element. This explains the relatively smooth velocity and dispersion maps in Figure 6, as we would expect more variation if only a small number of discrete absorbing clouds contributed to each spaxel (as discussed in Section 5.2). Our kinematic measurements thus most likely represent the collective range of motion of individual clouds within the line of sight toward each spaxel (with minimal contribution from the clouds' intrinsic line widths). We therefore interpret these high velocity dispersions, as well as the low velocity offsets, as arising from dispersion support on the scales probed within the CGM.

Our argument for a mainly dispersion-supported CGM in our observed system differs from other tomographic surveys of gravitational arcs (Lopez et al. 2018, 2020). The previous studies by Lopez et al. found evidence of a rotation signature in the CGM, suggesting that the observations probed accretion onto the absorber galaxy. However, as discussed in the previous section, our results favor a CGM supported largely by velocity dispersion. Since the galaxies studied by Lopez et al. are slightly less massive, and the inferred halo mass for our absorber galaxy is near the value where simulations predict a CGM phase transition from cold to hot (e.g., Kereš et al. 2005; Stern et al. 2020), the differences can plausibly arise in part from halo mass-dependent effects. Our results highlight that velocity dispersion, in addition to bulk velocity, is an important parameter for understanding CGM kinematics.

We also consider the possibility of an underlying subdominant outflow component contributing to the dispersion support in the CGM. Our triple Gaussian fits for both arcs showed apparent underestimates in the cleaner $\mathrm{Mg}$ II $\lambda 2803$ line (Figure 10(a)). The residual absorption is at redshifted velocities in arc 1 and blueshifted in arc 2, consistent with the distinct velocity offset between the arcs (Figure 9). If this residual absorption were to originate from a biconical outflow component in the CGM, we would expect an improvement in our fits by searching for an underlying broad secondary component in addition to the dominant $\sigma \approx 50 \mathrm{~km} \mathrm{~s}^{-1}$ CGM component. To test whether such an additional kinematic component is present, we fit the redder $\mathrm{Mg}$ II line with a double Gaussian profile: a narrow component corresponding to dispersion-dominated gas and a broader outflow component. We do not consider the $\lambda 2796$ line due to blending with strong Si II $\lambda 1260$ in the background arcs. We applied the fit to both arcs and compared the results with the galaxy in Figure 10(b).

The absorber galaxy has a significant improvement with the addition of the outflow component in its down-the-barrel spectrum (corresponding to a $5.5 \sigma$ detection of a second component), illustrating that the absorption profile is intrinsically non-Gaussian. This is consistent with previous observations of outflows driven by stellar feedback from galactic disks, where down-the-barrel absorption profiles are typically asymmetric with a broad tail toward blueshifted velocities (e.g., Bouché et al. 2012; Bordoloi et al. 2014; Rubin et al. 2014; Schroetter et al. 2016). The narrow component of the fit is centered near the estimated systemic velocity and may represent the galaxy's interstellar medium, while the broader blueshifted component is clearly associated with outflowing gas detected out to roughly $-500 \mathrm{~km} \mathrm{~s}^{-1}$. The arcs, on the other hand, are characterized by a dominant narrow absorption profile with relatively little contribution from a broader outflow component. Arc 2 does experience a significant improvement when an outflow component is added (corresponding to a $4.4 \sigma$ detection of a second component), whereas arc 1 shows no detectable improvement $(0.6 \sigma)$. The lack of outflow detection in arc 1 may be due in part to the lower $\mathrm{S} / \mathrm{N}$ of its spectrum compared to arc 2 and the generally lower $\mathrm{Mg}$ II equivalent width corresponding to larger impact parameters. We conclude that there is statistically significant evidence of outflowing gas along the line of sight in at least some directions toward the background arcs, although the total absorption profile in the arcs is dominated by a $\sigma \approx 50 \mathrm{~km} \mathrm{~s}^{-1}$ component centered near the systemic velocity.

To determine the extent to which the outflow is detected in arc 2, we separately examined two halves of the arc corresponding to impact parameters of $\sim 12$ and $\sim 19 \mathrm{kpc}$. We found that a similar outflow signature was present at both impact parameters (with $5.0 \sigma$ and $3.0 \sigma$ significance, respectively). Therefore, it appears as though the outflow extends 


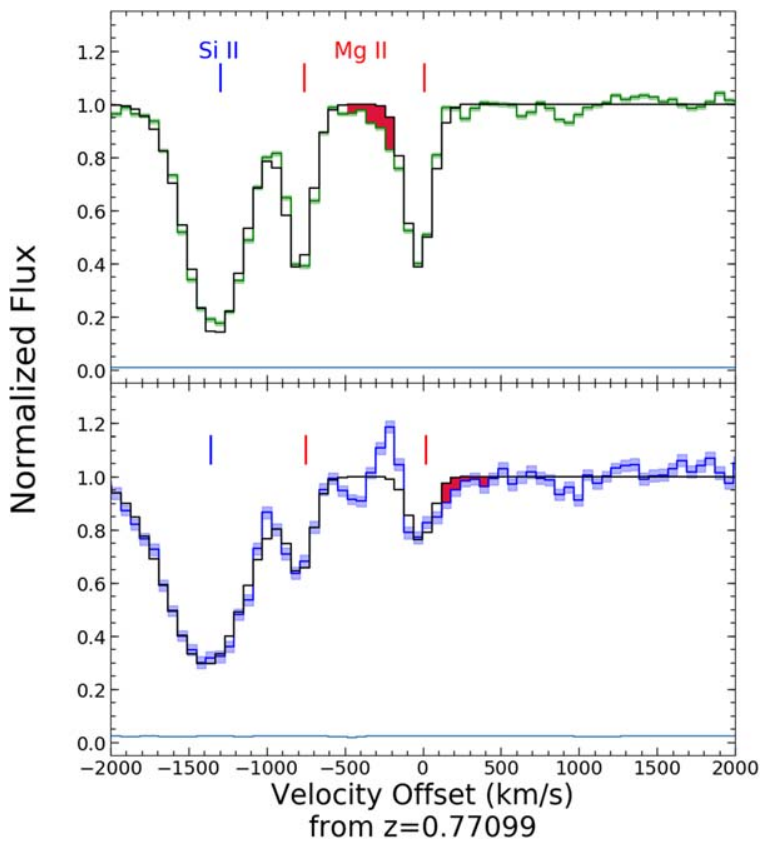

(a)

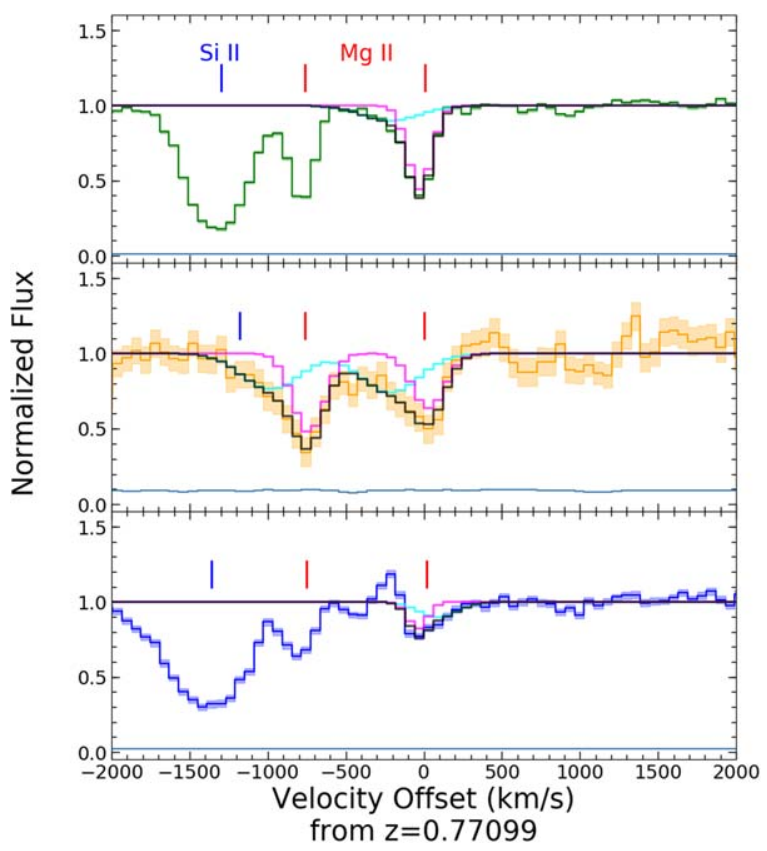

(b)

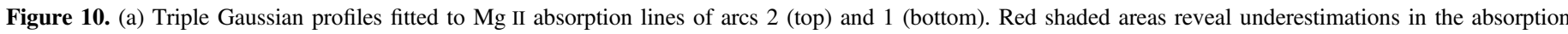

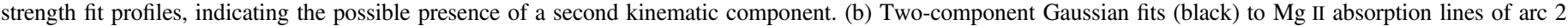

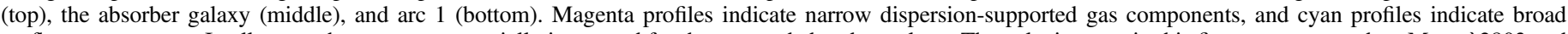

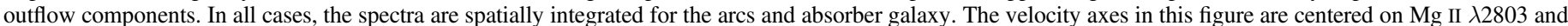
not $\mathrm{Mg}$ II $\lambda 2796$; we do not fit the $\lambda 2796$ line in the background arcs due to blending with the arcs' strong Si II $\lambda 1260$ feature.

throughout the entirety of arc 2. However, the outflow is overpowered by the dispersion-supported CGM. The presence of a prominent metal-enriched outflow in down-the-barrel absorption may trace "recycling" winds at relatively small impact parameters from the central galaxy (e.g., Oppenheimer et al. 2010; Hafen et al. 2019).

It is interesting to consider the effects of azimuthal sampling. The gravitational lens model reconstruction (Section 3 and Figure 3) indicates that the two arcs are roughly aligned with the minor axes of the absorber galaxy and do not sample along the major axis (Figure 5). Other studies have examined CGM absorption dependence on azimuthal angle (Bordoloi et al. 2011; Lan \& Mo 2018; Martin et al. 2019), with typically large equivalent widths toward the minor axis where we expect gas outflows to be more prominent in star-forming disk galaxies (Veilleux et al. 2005). If the strong absorption we see along the minor axis is indicative of outflows, as other works suggest (e.g., Bordoloi et al. 2011; Bouché et al. 2012; Kacprzak et al. 2012; Lan et al. 2014; Lan \& Mo 2018), one would expect the outflowing gas to have a velocity offset and a possibly large velocity dispersion. While the arcs show a relatively small velocity offset with high dispersions, the velocity dispersions in the arcs overall are much smaller than the clear outflow component seen in the absorber galaxy spectrum (Figure 8; Table 4), indicative of a subdominant outflowing gas component consistent with the two-component analysis discussed earlier.

To examine the extent to which this secondary outflow varies azimuthally, we performed the same two-component analysis on the regions of arc 2 previously configured in Figure 8. The analysis indicates that the outflow component appears fairly consistent throughout the four regions $(2.6 \sigma$, $2.6 \sigma, 5.2 \sigma$, and $3.8 \sigma$ significance, respectively; see Table 4 for reference), with loss of significance likely contributed by radial variation rather than azimuthal variation. Although we formally detect this outflow component in only one arc, we reiterate that arc 1 corresponds to larger impact parameters and has a lower continuum $\mathrm{S} / \mathrm{N}$. Based on the regions of arc 2 with comparable $D$, we would expect $\lesssim 1.5 \sigma$ significance of a comparable broad component for each binned region of arc 1 (as shown in Figure 8), such that the nondetections are not constraining. We thus view the broad component in arc 2 as likely indicative of a (subdominant) biconical outflow of metal-enriched gas into the CGM, with detection across the azimuthal extent of arc 2 indicating at least a moderately wide opening angle.

Some of the enriched CGM gas may be accreting onto the galaxy, rather than outflowing. The prevalence of cool, metalenriched gas with modest velocity suggests that recycling gas replenishes the CGM and may provide a reservoir to support ongoing star formation (possibly via spiraling inward near the disk plane; e.g., Ho et al. 2017; Martin et al. 2019). However, we cannot draw robust conclusions on inflows from the CGM based on our results. To confirm whether $\mathrm{Mg}$ II absorption may trace a bulk inflowing gas component, we would require measurements of the absorber galaxy rotation curve to model the expected CGM velocity field. Obtaining kinematic measurements of the absorber galaxy is therefore promising for further constraining inflows and outflows in this system.

\section{Discussion and Conclusions}

The CGM is important for understanding the gas flow processes that drive the evolution of galaxies. To better understand the CGM spatial structure and kinematics 
Table 5

Extended Data Table of Mg II Absorption Distribution and Kinematics

\begin{tabular}{|c|c|c|c|c|c|c|c|}
\hline $\begin{array}{l}\Delta \alpha^{\mathrm{a}} \\
(\mathrm{kpc})\end{array}$ & $\begin{array}{c}\Delta \delta^{\mathrm{a}} \\
(\mathrm{kpc})\end{array}$ & $\begin{array}{c}D^{\mathrm{b}} \\
(\mathrm{kpc})\end{array}$ & $\begin{array}{l}W_{0}{ }^{\mathrm{c}} \\
(\AA)\end{array}$ & $\begin{array}{c}v^{\mathrm{d}} \\
\left(\mathrm{km} \mathrm{s}^{-1}\right)\end{array}$ & $\begin{array}{c}\sigma^{\mathrm{e}} \\
\left(\mathrm{km} \mathrm{s}^{-1}\right)\end{array}$ & $\overline{\Sigma_{M g}{ }^{f}}$ & $\overline{\mathrm{S} / \mathrm{N}^{\mathrm{g}}}$ \\
\hline-20.27 & -19.39 & 28.05 & $0.09 \pm 0.66$ & $-8.62 \pm 174.97$ & $157.38 \pm 173.04$ & 1.22 & 5.11 \\
\hline-19.02 & -19.39 & 27.16 & $0.35 \pm 0.27$ & $-2.45 \pm 32.07$ & $0.0 \pm 72.61$ & 1.61 & 5.47 \\
\hline-17.75 & -19.40 & 26.30 & $0.34 \pm 0.28$ & $30.09 \pm 27.36$ & $26.07 \pm 46.9$ & 2.80 & 5.88 \\
\hline-16.49 & -19.42 & 25.48 & $0.4 \pm 0.27$ & $55.59 \pm 20.11$ & $27.01 \pm 35.89$ & 4.20 & 5.68 \\
\hline-15.22 & -19.45 & 24.70 & $0.59 \pm 0.28$ & $53.43 \pm 17.47$ & $18.33 \pm 36.57$ & 4.52 & 5.59 \\
\hline-13.95 & -19.50 & 23.97 & $0.9 \pm 0.3$ & $44.81 \pm 17.69$ & $21.99 \pm 34.96$ & 4.98 & 5.38 \\
\hline-12.67 & -19.56 & 23.30 & $0.86 \pm 0.28$ & $54.35 \pm 16.26$ & $0.0 \pm 48.87$ & 5.33 & 5.78 \\
\hline-11.38 & -19.62 & 22.69 & $0.66 \pm 0.27$ & $66.16 \pm 19.56$ & $0.0 \pm 54.52$ & 4.21 & 6.14 \\
\hline-10.09 & -19.70 & 22.14 & $0.41 \pm 0.26$ & $70.42 \pm 33.05$ & $0.0 \pm 74.6$ & 1.62 & 6.41 \\
\hline-8.79 & -19.79 & 21.66 & $0.14 \pm 0.21$ & $91.64 \pm 40.7$ & $0.0 \pm 82.61$ & $\ldots$ & 6.42 \\
\hline-7.47 & -19.89 & 21.25 & $0.22 \pm 0.19$ & $68.61 \pm 20.65$ & $0.0 \pm 54.54$ & 3.05 & 5.74 \\
\hline-26.49 & -17.75 & 31.89 & $0.44 \pm 0.25$ & $-80.05 \pm 36.43$ & $0.0 \pm 77.75$ & 0.93 & 5.05 \\
\hline-25.24 & -17.69 & 30.82 & $0.46 \pm 0.23$ & $-49.56 \pm 28.02$ & $0.0 \pm 67.31$ & 2.31 & 5.93 \\
\hline-24.00 & -17.63 & 29.78 & $0.34 \pm 0.23$ & $-20.05 \pm 30.82$ & $0.0 \pm 70.84$ & 1.97 & 6.58 \\
\hline-22.75 & -17.59 & 28.76 & $0.85 \pm 1.01$ & $-170.38 \pm 160.91$ & $279.6 \pm 125.73$ & 4.09 & 6.96 \\
\hline-21.51 & -17.57 & 27.77 & $0.76 \pm 0.63$ & $-156.59 \pm 75.75$ & $160.88 \pm 48.77$ & 4.50 & 6.84 \\
\hline-20.26 & -17.55 & 26.81 & $0.11 \pm 0.22$ & $-91.94 \pm 35.21$ & $0.0 \pm 80.91$ & 2.96 & 6.77 \\
\hline-19.02 & -17.55 & 25.88 & $0.38 \pm 0.23$ & $-32.61 \pm 21.39$ & $0.0 \pm 57.99$ & 3.43 & 6.97 \\
\hline-17.77 & -17.56 & 24.98 & $0.48 \pm 0.23$ & $0.15 \pm 17.59$ & $0.0 \pm 50.9$ & 4.75 & 7.11 \\
\hline-16.52 & -17.58 & 24.13 & $0.57 \pm 0.23$ & $21.37 \pm 14.45$ & $15.41 \pm 33.31$ & 5.99 & 6.72 \\
\hline-15.26 & -17.62 & 23.31 & $0.9 \pm 0.23$ & $17.74 \pm 12.37$ & $3.19 \pm 38.77$ & 6.53 & 6.47 \\
\hline-14.00 & -17.67 & 22.54 & $1.03 \pm 0.23$ & $23.71 \pm 12.66$ & $0.0 \pm 42.12$ & 6.52 & 6.49 \\
\hline-12.73 & -17.72 & 21.82 & $1.05 \pm 0.24$ & $41.18 \pm 14.75$ & $33.6 \pm 25.54$ & 6.65 & 6.91 \\
\hline-11.46 & -17.79 & 21.17 & $1.0 \pm 0.28$ & $53.33 \pm 19.69$ & $61.7 \pm 26.76$ & 6.29 & 7.41 \\
\hline-10.18 & -17.88 & 20.57 & $0.75 \pm 0.24$ & $68.04 \pm 20.59$ & $61.42 \pm 27.81$ & 5.25 & 7.84 \\
\hline-8.88 & -17.97 & 20.04 & $0.57 \pm 0.2$ & $65.01 \pm 18.63$ & $55.34 \pm 25.61$ & 5.24 & 7.93 \\
\hline-7.58 & -18.07 & 19.60 & $0.47 \pm 0.17$ & $50.55 \pm 14.63$ & $43.37 \pm 22.29$ & 5.97 & 7.38 \\
\hline-6.26 & -18.18 & 19.23 & $0.49 \pm 0.18$ & $44.67 \pm 15.05$ & $41.16 \pm 23.35$ & 5.68 & 6.63 \\
\hline-4.94 & -18.30 & 18.96 & $0.7 \pm 0.21$ & $28.3 \pm 17.24$ & $36.1 \pm 28.0$ & 4.68 & 5.99 \\
\hline-3.60 & -18.43 & 18.78 & $0.9 \pm 0.26$ & $9.52 \pm 19.77$ & $34.1 \pm 32.55$ & 4.17 & 5.53 \\
\hline-2.24 & -18.57 & 18.71 & $0.88 \pm 0.26$ & $26.44 \pm 20.4$ & $25.49 \pm 36.31$ & 3.73 & 5.26 \\
\hline-27.66 & -16.00 & 31.95 & $0.06 \pm 0.86$ & $184.63 \pm 562.58$ & $365.39 \pm 521.57$ & $\ldots$ & 5.74 \\
\hline-26.42 & -15.92 & 30.85 & $0.39 \pm 0.19$ & $-26.11 \pm 32.1$ & $0.0 \pm 71.24$ & 1.79 & 7.78 \\
\hline-25.18 & -15.86 & 29.76 & $0.46 \pm 0.18$ & $2.88 \pm 30.34$ & $37.13 \pm 45.1$ & 2.83 & 9.11 \\
\hline-23.95 & -15.80 & 28.70 & $0.47 \pm 0.19$ & $-13.71 \pm 31.77$ & $48.6 \pm 43.97$ & 3.25 & 9.54 \\
\hline-22.72 & -15.76 & 27.65 & $0.44 \pm 0.22$ & $-57.37 \pm 28.95$ & $38.65 \pm 45.5$ & 3.62 & 9.39 \\
\hline-21.49 & -15.74 & 26.63 & $0.41 \pm 0.21$ & $-80.62 \pm 22.64$ & $0.0 \pm 61.5$ & 4.23 & 8.81 \\
\hline-20.26 & -15.72 & 25.64 & $0.36 \pm 0.21$ & $-74.23 \pm 20.09$ & $0.0 \pm 57.93$ & 4.44 & 8.34 \\
\hline-19.02 & -15.72 & 24.68 & $0.51 \pm 0.22$ & $-44.47 \pm 16.27$ & $0.0 \pm 49.91$ & 5.29 & 7.76 \\
\hline-17.79 & -15.73 & 23.75 & $0.75 \pm 0.22$ & $-16.47 \pm 12.74$ & $0.0 \pm 42.53$ & 6.82 & 7.21 \\
\hline-16.55 & -15.76 & 22.85 & $0.91 \pm 0.21$ & $-6.76 \pm 10.92$ & $0.0 \pm 38.84$ & 7.33 & 6.51 \\
\hline-15.31 & -15.79 & 21.99 & $1.09 \pm 0.22$ & $-5.21 \pm 11.37$ & $0.0 \pm 39.5$ & 6.81 & 6.19 \\
\hline-14.06 & -15.84 & 21.18 & $1.07 \pm 0.22$ & $5.2 \pm 12.37$ & $0.0 \pm 41.43$ & 6.17 & 6.13 \\
\hline-12.80 & -15.90 & 20.42 & $1.1 \pm 0.24$ & $19.34 \pm 14.78$ & $43.64 \pm 23.15$ & 6.34 & 6.66 \\
\hline-11.54 & -15.98 & 19.71 & $1.5 \pm 0.3$ & $34.7 \pm 19.7$ & $96.62 \pm 23.38$ & 8.05 & 7.16 \\
\hline-10.26 & -16.06 & 19.06 & $1.36 \pm 0.25$ & $45.26 \pm 15.39$ & $86.51 \pm 18.91$ & 8.99 & 7.75 \\
\hline-8.98 & -16.16 & 18.48 & $1.01 \pm 0.21$ & $50.29 \pm 14.91$ & $79.48 \pm 18.57$ & 8.43 & 7.81 \\
\hline-7.69 & -16.26 & 17.99 & $0.62 \pm 0.2$ & $45.86 \pm 17.27$ & $71.63 \pm 22.0$ & 7.08 & 7.54 \\
\hline-6.38 & -16.38 & 17.58 & $0.58 \pm 0.21$ & $44.14 \pm 19.72$ & $64.23 \pm 25.66$ & 6.21 & 7.11 \\
\hline-5.06 & -16.50 & 17.26 & $0.82 \pm 0.23$ & $43.45 \pm 19.05$ & $55.66 \pm 25.93$ & 5.97 & 6.65 \\
\hline-3.73 & -16.63 & 17.05 & $0.91 \pm 0.23$ & $42.75 \pm 18.66$ & $47.84 \pm 26.81$ & 5.55 & 6.40 \\
\hline-2.39 & -16.77 & 16.94 & $0.88 \pm 0.21$ & $54.21 \pm 15.88$ & $37.12 \pm 25.46$ & 5.63 & 6.50 \\
\hline-1.03 & -16.92 & 16.95 & $0.88 \pm 0.18$ & $62.28 \pm 11.6$ & $33.6 \pm 20.18$ & 7.36 & 6.40 \\
\hline 0.35 & -17.07 & 17.08 & $0.9 \pm 0.2$ & $66.59 \pm 11.12$ & $43.07 \pm 17.39$ & 8.87 & 6.60 \\
\hline 1.74 & -17.24 & 17.32 & $0.87 \pm 0.22$ & $61.14 \pm 11.99$ & $44.56 \pm 18.53$ & 8.51 & 6.87 \\
\hline 3.15 & -17.40 & 17.68 & $1.04 \pm 0.21$ & $47.72 \pm 11.58$ & $40.26 \pm 18.85$ & 7.73 & 6.72 \\
\hline 4.57 & -17.57 & 18.16 & $1.17 \pm 0.26$ & $61.59 \pm 17.49$ & $64.79 \pm 22.68$ & 6.47 & 5.85 \\
\hline-27.57 & -14.19 & 31.01 & $0.4 \pm 0.17$ & $-7.74 \pm 30.8$ & $0.0 \pm 68.87$ & 1.64 & 7.47 \\
\hline-26.35 & -14.11 & 29.89 & $0.5 \pm 0.31$ & $184.63 \pm 151.11$ & $221.04 \pm 149.03$ & 2.96 & 9.91 \\
\hline-25.12 & -14.04 & 28.78 & $0.6 \pm 0.33$ & $184.63 \pm 141.18$ & $226.64 \pm 138.12$ & 3.88 & 11.52 \\
\hline-23.90 & -13.99 & 27.70 & $0.4 \pm 0.16$ & $-51.08 \pm 27.5$ & $33.88 \pm 43.81$ & 3.89 & 11.67 \\
\hline-22.69 & -13.95 & 26.63 & $0.51 \pm 0.18$ & $-75.89 \pm 19.85$ & $20.27 \pm 39.87$ & 5.27 & 10.88 \\
\hline-21.47 & -13.92 & 25.59 & $0.49 \pm 0.2$ & $-84.95 \pm 20.31$ & $0.0 \pm 56.99$ & 5.01 & 9.67 \\
\hline
\end{tabular}


Table 5

(Continued)

\begin{tabular}{|c|c|c|c|c|c|c|c|}
\hline $\begin{array}{l}\Delta \alpha^{\mathrm{a}} \\
(\mathrm{kpc})\end{array}$ & $\begin{array}{c}\Delta \delta^{\mathrm{a}} \\
(\mathrm{kpc})\end{array}$ & $\begin{array}{c}D^{\mathrm{b}} \\
(\mathrm{kpc})\end{array}$ & $\begin{array}{l}W_{0}^{\mathrm{c}} \\
(\AA)\end{array}$ & $\begin{array}{c}v^{\mathrm{d}} \\
\left(\mathrm{km} \mathrm{s}^{-1}\right)\end{array}$ & $\begin{array}{c}\sigma^{\mathrm{e}} \\
\left(\mathrm{km} \mathrm{s}^{-1}\right)\end{array}$ & $\Sigma_{\mathrm{Mg}}^{\mathrm{f}}$ & $\mathrm{S} / \mathrm{N}^{\mathrm{g}}$ \\
\hline-20.25 & -13.91 & 24.56 & $0.49 \pm 0.21$ & $-73.39 \pm 18.1$ & $0.0 \pm 53.93$ & 5.01 & 8.43 \\
\hline-19.03 & -13.91 & 23.57 & $0.71 \pm 0.22$ & $-43.33 \pm 13.57$ & $0.0 \pm 44.71$ & 6.45 & 7.16 \\
\hline-17.81 & -13.92 & 22.60 & $1.06 \pm 0.23$ & $-20.19 \pm 11.38$ & $24.47 \pm 23.5$ & 7.96 & 6.28 \\
\hline-16.58 & -13.94 & 21.66 & $1.1 \pm 0.23$ & $-18.86 \pm 11.41$ & $0.01 \pm 39.57$ & 6.88 & 5.58 \\
\hline-12.87 & -14.10 & 19.09 & $0.96 \pm 0.29$ & $17.59 \pm 19.36$ & $41.51 \pm 30.8$ & 4.20 & 5.27 \\
\hline-11.62 & -14.17 & 18.32 & $1.87 \pm 0.38$ & $18.05 \pm 29.37$ & $135.97 \pm 30.81$ & 6.72 & 5.73 \\
\hline-10.35 & -14.26 & 17.62 & $1.46 \pm 0.28$ & $34.47 \pm 16.91$ & $88.92 \pm 20.7$ & 7.83 & 6.16 \\
\hline-9.08 & -14.35 & 16.99 & $1.1 \pm 0.25$ & $39.49 \pm 17.42$ & $79.55 \pm 21.71$ & 7.01 & 6.29 \\
\hline-7.80 & -14.46 & 16.43 & $0.68 \pm 0.28$ & $55.96 \pm 31.33$ & $100.53 \pm 36.75$ & 4.72 & 6.29 \\
\hline-6.50 & -14.58 & 15.96 & $0.65 \pm 0.3$ & $58.39 \pm 35.76$ & $92.56 \pm 41.87$ & 4.25 & 6.16 \\
\hline-5.19 & -14.71 & 15.60 & $0.81 \pm 0.27$ & $45.17 \pm 23.75$ & $62.33 \pm 30.72$ & 5.32 & 6.10 \\
\hline-3.87 & -14.84 & 15.34 & $0.87 \pm 0.24$ & $52.89 \pm 18.63$ & $53.93 \pm 25.56$ & 5.93 & 6.21 \\
\hline-2.53 & -14.98 & 15.20 & $0.92 \pm 0.2$ & $58.54 \pm 13.78$ & $44.53 \pm 20.82$ & 6.92 & 6.59 \\
\hline-1.18 & -15.13 & 15.18 & $1.08 \pm 0.19$ & $59.13 \pm 11.23$ & $46.71 \pm 16.88$ & 9.09 & 6.96 \\
\hline 0.19 & -15.29 & 15.29 & $1.16 \pm 0.19$ & $57.09 \pm 10.65$ & $52.69 \pm 15.29$ & 10.50 & 7.47 \\
\hline 1.58 & -15.46 & 15.54 & $1.04 \pm 0.18$ & $50.52 \pm 10.18$ & $45.25 \pm 15.87$ & 10.31 & 8.16 \\
\hline 2.98 & -15.62 & 15.91 & $1.17 \pm 0.2$ & $52.22 \pm 10.67$ & $52.81 \pm 15.56$ & 10.33 & 8.44 \\
\hline 4.40 & -15.80 & 16.40 & $1.57 \pm 0.26$ & $73.5 \pm 16.48$ & $93.37 \pm 19.37$ & 9.86 & 7.65 \\
\hline 5.83 & -15.98 & 17.01 & $1.37 \pm 0.3$ & $65.14 \pm 19.49$ & $75.49 \pm 24.09$ & 7.25 & 6.33 \\
\hline 7.29 & -16.16 & 17.73 & $1.07 \pm 0.31$ & $51.16 \pm 21.14$ & $44.8 \pm 31.12$ & 4.48 & 5.05 \\
\hline-28.70 & -12.48 & 31.30 & $0.37 \pm 0.23$ & $0.43 \pm 43.9$ & $0.0 \pm 85.72$ & $\ldots$ & 5.82 \\
\hline-27.49 & -12.38 & 30.15 & $0.37 \pm 0.16$ & $6.66 \pm 30.52$ & $0.0 \pm 68.81$ & 1.58 & 8.14 \\
\hline-26.27 & -12.30 & 29.01 & $0.45 \pm 0.32$ & $184.63 \pm 145.61$ & $189.79 \pm 178.76$ & 2.02 & 10.43 \\
\hline-25.06 & -12.23 & 27.89 & $0.46 \pm 0.29$ & $184.63 \pm 137.74$ & $200.48 \pm 148.99$ & 2.76 & 11.66 \\
\hline-23.86 & -12.18 & 26.79 & $0.38 \pm 0.15$ & $-76.85 \pm 22.13$ & $0.0 \pm 58.87$ & 4.42 & 11.48 \\
\hline-22.65 & -12.14 & 25.70 & $0.54 \pm 0.16$ & $-93.65 \pm 17.32$ & $0.0 \pm 50.82$ & 5.78 & 10.19 \\
\hline-21.45 & -12.11 & 24.63 & $0.55 \pm 0.2$ & $-101.55 \pm 19.68$ & $0.0 \pm 54.45$ & 5.35 & 8.56 \\
\hline-20.24 & -12.10 & 23.58 & $0.55 \pm 0.22$ & $-92.37 \pm 20.21$ & $0.0 \pm 56.57$ & 4.56 & 7.12 \\
\hline-19.04 & -12.10 & 22.56 & $0.83 \pm 0.24$ & $-54.58 \pm 18.72$ & $38.72 \pm 30.66$ & 4.81 & 5.86 \\
\hline-2.68 & -13.21 & 13.48 & $0.92 \pm 0.23$ & $51.43 \pm 15.78$ & $41.45 \pm 24.27$ & 5.79 & 5.47 \\
\hline-1.33 & -13.36 & 13.43 & $1.15 \pm 0.22$ & $52.58 \pm 14.36$ & $51.15 \pm 20.43$ & 7.34 & 6.13 \\
\hline 0.03 & -13.52 & 13.52 & $1.22 \pm 0.2$ & $49.78 \pm 13.5$ & $60.89 \pm 18.09$ & 8.27 & 6.97 \\
\hline 1.41 & -13.69 & 13.76 & $1.2 \pm 0.2$ & $56.98 \pm 13.41$ & $72.22 \pm 17.07$ & 8.91 & 7.60 \\
\hline 2.81 & -13.86 & 14.14 & $1.66 \pm 0.23$ & $89.4 \pm 16.06$ & $111.07 \pm 18.35$ & 10.50 & 8.23 \\
\hline 4.22 & -14.03 & 14.65 & $1.79 \pm 0.25$ & $83.64 \pm 17.13$ & $111.91 \pm 19.51$ & 10.45 & 7.80 \\
\hline 5.65 & -14.21 & 15.30 & $1.42 \pm 0.24$ & $49.2 \pm 15.37$ & $63.78 \pm 20.16$ & 8.09 & 6.84 \\
\hline 7.10 & -14.40 & 16.05 & $1.11 \pm 0.24$ & $40.86 \pm 16.28$ & $33.89 \pm 27.06$ & 5.25 & 5.88 \\
\hline 8.57 & -14.58 & 16.92 & $0.68 \pm 0.26$ & $61.54 \pm 25.35$ & $18.03 \pm 46.9$ & 2.40 & 5.01 \\
\hline-28.60 & -10.69 & 30.53 & $0.39 \pm 0.74$ & $-186.18 \pm 276.28$ & $120.71 \pm 253.41$ & $\ldots$ & 5.67 \\
\hline-27.40 & -10.59 & 29.37 & $0.37 \pm 3.58$ & $-186.02 \pm 2740.28$ & $257.12 \pm 1630.5$ & $\ldots$ & 7.45 \\
\hline-26.20 & -10.51 & 28.23 & $0.36 \pm 0.32$ & $184.63 \pm 147.98$ & $153.93 \pm 193.14$ & 0.83 & 8.98 \\
\hline-25.00 & -10.44 & 27.09 & $0.85 \pm 1.05$ & $-186.18 \pm 1003.32$ & $365.85 \pm 352.21$ & 2.85 & 9.54 \\
\hline-23.81 & -10.39 & 25.98 & $0.37 \pm 0.17$ & $-102.85 \pm 24.35$ & $0.0 \pm 61.02$ & 4.35 & 9.05 \\
\hline-22.62 & -10.35 & 24.87 & $0.54 \pm 0.18$ & $-111.81 \pm 20.5$ & $0.0 \pm 53.71$ & 5.17 & 7.91 \\
\hline-21.43 & -10.32 & 23.78 & $0.6 \pm 0.23$ & $-108.09 \pm 21.84$ & $0.0 \pm 56.63$ & 4.52 & 6.36 \\
\hline-20.24 & -10.31 & 22.71 & $0.6 \pm 0.26$ & $-107.65 \pm 25.76$ & $0.0 \pm 62.23$ & 3.18 & 5.12 \\
\hline-0.14 & -11.76 & 11.76 & $0.9 \pm 0.21$ & $13.35 \pm 14.7$ & $0.0 \pm 45.25$ & 4.53 & 5.30 \\
\hline 1.24 & -11.93 & 11.99 & $1.45 \pm 0.3$ & $85.75 \pm 28.01$ & $127.51 \pm 31.65$ & 5.99 & 5.89 \\
\hline 2.63 & -12.10 & 12.39 & $1.63 \pm 0.28$ & $96.58 \pm 22.12$ & $128.98 \pm 25.18$ & 7.70 & 6.40 \\
\hline 4.04 & -12.28 & 12.93 & $1.15 \pm 0.23$ & $59.32 \pm 14.48$ & $63.75 \pm 19.21$ & 7.24 & 6.55 \\
\hline 5.47 & -12.46 & 13.61 & $0.89 \pm 0.24$ & $55.54 \pm 16.96$ & $33.19 \pm 28.49$ & 5.27 & 6.22 \\
\hline 6.92 & -12.65 & 14.42 & $0.59 \pm 0.23$ & $80.13 \pm 25.28$ & $22.97 \pm 44.17$ & 2.76 & 5.90 \\
\hline 8.38 & -12.84 & 15.33 & $0.0 \pm 0.21$ & $165.99 \pm 29.04$ & $46.98 \pm 39.96$ & 2.31 & 5.28 \\
\hline-28.50 & -8.91 & 29.86 & $0.12 \pm 1.06$ & $-186.18 \pm 974.14$ & $108.78 \pm 764.66$ & $\ldots$ & 5.14 \\
\hline-27.31 & -8.81 & 28.69 & $0.33 \pm 7.39$ & $-186.18 \pm 6803.49$ & $288.17 \pm 3672.24$ & $\ldots$ & 6.12 \\
\hline-26.12 & -8.73 & 27.54 & $0.6 \pm 0.63$ & $184.63 \pm 202.72$ & $206.69 \pm 281.61$ & 1.62 & 6.67 \\
\hline-24.94 & -8.66 & 26.40 & $1.1 \pm 0.67$ & $-186.18 \pm 176.53$ & $219.25 \pm 80.39$ & 3.89 & 6.56 \\
\hline-23.76 & -8.60 & 25.27 & $0.5 \pm 0.22$ & $-106.56 \pm 23.07$ & $0.0 \pm 58.59$ & 3.96 & 6.15 \\
\hline-22.58 & -8.56 & 24.15 & $0.5 \pm 0.27$ & $-113.12 \pm 28.09$ & $0.0 \pm 64.52$ & 3.39 & 5.22 \\
\hline 6.73 & -10.91 & 12.82 & $0.04 \pm 0.24$ & $154.39 \pm 33.89$ & $46.14 \pm 46.05$ & 2.06 & 5.23 \\
\hline 8.19 & -11.10 & 13.80 & $0.0 \pm 0.23$ & $157.22 \pm 29.67$ & $61.35 \pm 37.77$ & 2.70 & 5.14 \\
\hline-2.32 & -2.99 & 3.78 & $2.06 \pm 0.45$ & $36.27 \pm 45.45$ & $164.81 \pm 41.92$ & 5.53 & 5.09 \\
\hline-0.99 & -3.16 & 3.31 & $2.26 \pm 0.72$ & $18.46 \pm 37.74$ & $154.21 \pm 35.4$ & 6.93 & 5.73 \\
\hline
\end{tabular}


Table 5

(Continued)

\begin{tabular}{|c|c|c|c|c|c|c|c|}
\hline $\begin{array}{l}\Delta \alpha^{\mathrm{a}} \\
(\mathrm{kpc})\end{array}$ & $\begin{array}{c}\Delta \delta^{\mathrm{a}} \\
(\mathrm{kpc})\end{array}$ & $\begin{array}{c}D^{\mathrm{b}} \\
(\mathrm{kpc})\end{array}$ & $\begin{array}{l}W_{0}^{\mathrm{c}} \\
(\AA)\end{array}$ & $\begin{array}{c}v^{\mathrm{d}} \\
\left(\mathrm{km} \mathrm{s}^{-1}\right)\end{array}$ & $\begin{array}{c}\sigma^{\mathrm{e}} \\
\left(\mathrm{km} \mathrm{s}^{-1}\right)\end{array}$ & $\Sigma_{\mathrm{Mg}}^{\mathrm{f}}$ & $\mathrm{S} / \mathrm{N}^{\mathrm{g}}$ \\
\hline 0.36 & -3.34 & 3.36 & $2.17 \pm 0.35$ & $27.62 \pm 30.34$ & $152.51 \pm 28.97$ & 6.66 & 5.70 \\
\hline 1.73 & -3.52 & 3.92 & $1.47 \pm 0.36$ & $49.72 \pm 37.76$ & $129.76 \pm 38.77$ & 4.31 & 5.06 \\
\hline-3.79 & -1.14 & 3.95 & $2.47 \pm 0.44$ & $9.54 \pm 50.65$ & $204.33 \pm 35.09$ & 7.65 & 5.62 \\
\hline-2.49 & -1.31 & 2.81 & $2.59 \pm 0.34$ & $23.23 \pm 29.41$ & $177.13 \pm 24.41$ & 9.85 & 6.63 \\
\hline-1.16 & -1.48 & 1.89 & $2.46 \pm 0.5$ & $24.5 \pm 23.07$ & $151.98 \pm 21.71$ & 11.12 & 7.41 \\
\hline 0.18 & -1.66 & 1.67 & $2.28 \pm 0.47$ & $32.6 \pm 22.68$ & $145.31 \pm 22.3$ & 10.74 & 7.36 \\
\hline 1.55 & -1.84 & 2.41 & $1.86 \pm 0.48$ & $45.46 \pm 32.23$ & $132.66 \pm 27.74$ & 8.35 & 6.44 \\
\hline 2.93 & -2.03 & 3.57 & $1.21 \pm 0.39$ & $33.77 \pm 38.0$ & $109.12 \pm 40.44$ & 4.79 & 5.25 \\
\hline-3.95 & 0.52 & 3.99 & $2.99 \pm 0.42$ & $-6.39 \pm 47.54$ & $222.81 \pm 30.57$ & 10.32 & 6.29 \\
\hline-2.66 & 0.35 & 2.68 & $2.97 \pm 0.32$ & $15.38 \pm 28.52$ & $196.49 \pm 21.93$ & 12.55 & 7.36 \\
\hline-1.34 & 0.18 & 1.35 & $2.74 \pm 0.31$ & $18.32 \pm 22.46$ & $168.65 \pm 18.79$ & 13.99 & 8.17 \\
\hline 0.00 & 0.00 & 0.00 & $2.7 \pm 0.3$ & $15.73 \pm 21.07$ & $165.89 \pm 18.14$ & 14.28 & 8.20 \\
\hline 1.36 & -0.18 & 1.37 & $2.69 \pm 0.29$ & $8.55 \pm 21.77$ & $170.93 \pm 19.24$ & 12.70 & 7.18 \\
\hline 2.75 & -0.37 & 2.77 & $2.2 \pm 0.31$ & $-14.19 \pm 26.05$ & $158.32 \pm 24.11$ & 8.85 & 5.99 \\
\hline 4.15 & -0.56 & 4.19 & $1.46 \pm 0.41$ & $-71.27 \pm 43.86$ & $135.18 \pm 45.41$ & 4.54 & 5.26 \\
\hline-4.12 & 2.17 & 4.66 & $3.1 \pm 0.37$ & $21.45 \pm 38.74$ & $221.9 \pm 28.06$ & 10.28 & 5.77 \\
\hline-2.83 & 2.00 & 3.47 & $2.9 \pm 0.33$ & $19.85 \pm 27.93$ & $193.01 \pm 22.56$ & 11.85 & 6.82 \\
\hline-1.52 & 1.83 & 2.37 & $2.73 \pm 0.31$ & $8.34 \pm 22.81$ & $174.68 \pm 19.65$ & 13.13 & 7.60 \\
\hline-0.18 & 1.65 & 1.66 & $2.72 \pm 0.29$ & $1.43 \pm 20.02$ & $170.1 \pm 17.54$ & 13.79 & 7.54 \\
\hline 1.18 & 1.46 & 1.88 & $2.66 \pm 0.3$ & $-1.86 \pm 19.6$ & $159.09 \pm 18.19$ & 12.94 & 6.87 \\
\hline 2.56 & 1.28 & 2.86 & $2.14 \pm 0.33$ & $-7.67 \pm 20.56$ & $127.22 \pm 22.25$ & 9.85 & 5.89 \\
\hline 3.96 & 1.09 & 4.11 & $1.4 \pm 0.36$ & $-34.95 \pm 29.57$ & $110.37 \pm 33.62$ & 5.57 & 5.18 \\
\hline-3.00 & 3.63 & 4.71 & $2.32 \pm 0.4$ & $22.59 \pm 29.23$ & $156.62 \pm 27.26$ & 8.34 & 5.27 \\
\hline-1.69 & 3.46 & 3.85 & $2.11 \pm 0.38$ & $4.18 \pm 27.46$ & $147.42 \pm 26.48$ & 8.92 & 6.04 \\
\hline-0.36 & 3.28 & 3.30 & $2.06 \pm 0.33$ & $-13.38 \pm 23.62$ & $150.65 \pm 22.6$ & 9.72 & 6.13 \\
\hline 0.99 & 3.10 & 3.25 & $2.06 \pm 0.4$ & $-33.94 \pm 24.13$ & $150.46 \pm 22.07$ & 9.68 & 5.84 \\
\hline 2.37 & 2.91 & 3.75 & $1.71 \pm 0.37$ & $-35.0 \pm 25.12$ & $123.73 \pm 27.33$ & 7.40 & 5.41 \\
\hline-0.54 & 4.89 & 4.92 & $1.07 \pm 0.38$ & $-27.83 \pm 26.82$ & $109.67 \pm 29.41$ & 6.14 & 5.14 \\
\hline 0.81 & 4.71 & 4.78 & $0.37 \pm 0.45$ & $-62.42 \pm 21.87$ & $88.75 \pm 26.87$ & 5.62 & 5.03 \\
\hline-4.62 & 7.02 & 8.40 & $0.93 \pm 0.23$ & $28.83 \pm 13.88$ & $0.0 \pm 43.85$ & 5.05 & 5.06 \\
\hline-3.35 & 6.85 & 7.62 & $0.79 \pm 0.25$ & $12.41 \pm 18.35$ & $38.77 \pm 28.53$ & 4.94 & 5.47 \\
\hline-2.05 & 6.67 & 6.98 & $0.74 \pm 0.26$ & $-1.21 \pm 18.99$ & $61.85 \pm 24.84$ & 5.57 & 5.56 \\
\hline-0.72 & 6.49 & 6.53 & $0.87 \pm 0.28$ & $-15.61 \pm 19.73$ & $82.75 \pm 23.54$ & 6.14 & 5.56 \\
\hline 0.63 & 6.31 & 6.35 & $0.01 \pm 0.27$ & $-52.08 \pm 17.93$ & $47.25 \pm 25.97$ & 4.62 & 5.22 \\
\hline-7.26 & 8.92 & 11.50 & $0.9 \pm 0.21$ & $10.17 \pm 12.39$ & $26.95 \pm 23.65$ & 6.38 & 5.81 \\
\hline-6.04 & 8.76 & 10.64 & $1.07 \pm 0.19$ & $20.65 \pm 9.47$ & $14.9 \pm 24.31$ & 8.43 & 7.12 \\
\hline-4.79 & 8.59 & 9.84 & $1.0 \pm 0.17$ & $16.79 \pm 8.94$ & $0.0 \pm 34.7$ & 9.51 & 8.28 \\
\hline-3.52 & 8.42 & 9.13 & $0.93 \pm 0.16$ & $7.57 \pm 9.45$ & $26.8 \pm 18.89$ & 10.65 & 8.95 \\
\hline-2.22 & 8.25 & 8.55 & $1.04 \pm 0.17$ & $0.93 \pm 9.85$ & $47.86 \pm 14.86$ & 11.64 & 8.85 \\
\hline-0.90 & 8.08 & 8.13 & $1.2 \pm 0.18$ & $3.81 \pm 11.58$ & $74.61 \pm 14.57$ & 10.91 & 8.41 \\
\hline 0.45 & 7.90 & 7.91 & $1.45 \pm 0.27$ & $-4.45 \pm 21.11$ & $126.09 \pm 21.5$ & 8.93 & 7.42 \\
\hline 1.82 & 7.72 & 7.93 & $1.63 \pm 0.42$ & $-56.86 \pm 41.78$ & $170.17 \pm 30.83$ & 6.92 & 6.19 \\
\hline-8.61 & 10.64 & 13.69 & $0.82 \pm 0.19$ & $13.02 \pm 14.17$ & $30.92 \pm 24.97$ & 5.29 & 6.34 \\
\hline-7.42 & 10.48 & 12.84 & $1.06 \pm 0.14$ & $14.69 \pm 8.11$ & $34.56 \pm 14.57$ & 10.22 & 9.03 \\
\hline-6.20 & 10.32 & 12.04 & $1.06 \pm 0.13$ & $20.37 \pm 6.89$ & $25.2 \pm 14.95$ & 13.71 & 11.85 \\
\hline-4.96 & 10.15 & 11.30 & $1.05 \pm 0.12$ & $18.59 \pm 6.62$ & $21.74 \pm 15.58$ & 17.57 & 14.18 \\
\hline-3.69 & 9.98 & 10.65 & $1.13 \pm 0.12$ & $15.16 \pm 6.54$ & $35.4 \pm 11.84$ & 21.12 & 15.78 \\
\hline-2.40 & 9.81 & 10.10 & $1.25 \pm 0.12$ & $12.72 \pm 6.74$ & $49.54 \pm 10.22$ & 22.46 & 16.20 \\
\hline-1.08 & 9.64 & 9.70 & $1.31 \pm 0.14$ & $14.89 \pm 7.98$ & $64.47 \pm 10.7$ & 20.40 & 14.82 \\
\hline 0.27 & 9.47 & 9.47 & $1.31 \pm 0.17$ & $12.11 \pm 11.27$ & $86.11 \pm 13.52$ & 15.95 & 12.27 \\
\hline 1.64 & 9.29 & 9.43 & $1.43 \pm 0.25$ & $-17.79 \pm 18.61$ & $118.96 \pm 19.65$ & 11.89 & 9.53 \\
\hline 3.03 & 9.11 & 9.60 & $1.39 \pm 0.36$ & $-34.08 \pm 26.56$ & $125.69 \pm 26.57$ & 8.47 & 7.37 \\
\hline 4.44 & 8.93 & 9.97 & $0.0 \pm 0.58$ & $-33.66 \pm 38.47$ & $102.27 \pm 43.36$ & 3.58 & 5.31 \\
\hline-9.93 & 12.33 & 15.83 & $0.76 \pm 0.24$ & $64.43 \pm 22.46$ & $15.2 \pm 44.67$ & 2.87 & 5.36 \\
\hline-8.77 & 12.17 & 15.00 & $1.05 \pm 0.16$ & $21.52 \pm 10.83$ & $37.1 \pm 18.24$ & 8.26 & 8.62 \\
\hline-7.58 & 12.02 & 14.21 & $1.1 \pm 0.12$ & $15.08 \pm 7.76$ & $46.23 \pm 12.09$ & 14.33 & 13.00 \\
\hline-6.37 & 11.85 & 13.46 & $1.08 \pm 0.11$ & $16.6 \pm 6.66$ & $44.83 \pm 10.63$ & 20.30 & 17.74 \\
\hline-5.13 & 11.69 & 12.77 & $1.11 \pm 0.11$ & $18.66 \pm 6.03$ & $39.09 \pm 10.41$ & 27.15 & 21.99 \\
\hline-3.86 & 11.52 & 12.15 & $1.18 \pm 0.1$ & $18.59 \pm 5.7$ & $40.72 \pm 9.66$ & 33.15 & 25.27 \\
\hline-2.56 & 11.36 & 11.64 & $1.27 \pm 0.1$ & $15.68 \pm 5.79$ & $51.46 \pm 8.67$ & 35.87 & 26.28 \\
\hline-1.25 & 11.19 & 11.26 & $1.34 \pm 0.12$ & $13.85 \pm 6.73$ & $65.43 \pm 9.01$ & 34.00 & 24.21 \\
\hline 0.10 & 11.02 & 11.02 & $1.38 \pm 0.14$ & $11.88 \pm 8.2$ & $76.88 \pm 10.29$ & 28.14 & 19.87 \\
\hline 1.46 & 10.85 & 10.95 & $1.48 \pm 0.17$ & $2.11 \pm 10.37$ & $91.57 \pm 12.25$ & 21.62 & 15.11 \\
\hline
\end{tabular}


Table 5

(Continued)

\begin{tabular}{|c|c|c|c|c|c|c|c|}
\hline $\begin{array}{l}\Delta \alpha^{\mathrm{a}} \\
(\mathrm{kpc})\end{array}$ & $\begin{array}{c}\Delta \delta^{\mathrm{a}} \\
(\mathrm{kpc})\end{array}$ & $\begin{array}{c}D^{\mathrm{b}} \\
(\mathrm{kpc})\end{array}$ & $\begin{array}{l}W_{0}^{\mathrm{c}} \\
(\AA)\end{array}$ & $\begin{array}{c}v^{\mathrm{d}} \\
\left(\mathrm{km} \mathrm{s}^{-1}\right)\end{array}$ & $\begin{array}{c}\sigma^{\mathrm{e}} \\
\left(\mathrm{km} \mathrm{s}^{-1}\right)\end{array}$ & $\Sigma_{\mathrm{Mg}}^{\mathrm{f}}$ & $\mathrm{S} / \mathrm{N}^{\mathrm{g}}$ \\
\hline 2.85 & 10.67 & 11.05 & $1.52 \pm 0.22$ & $-8.68 \pm 13.19$ & $101.82 \pm 14.95$ & 15.80 & 10.90 \\
\hline 4.26 & 10.49 & 11.32 & $1.33 \pm 0.28$ & $-4.13 \pm 17.86$ & $103.21 \pm 19.91$ & 9.85 & 7.65 \\
\hline 5.70 & 10.31 & 11.78 & $0.87 \pm 0.4$ & $9.88 \pm 35.83$ & $105.7 \pm 39.01$ & 4.25 & 5.22 \\
\hline-10.08 & 13.84 & 17.12 & $0.8 \pm 0.21$ & $42.71 \pm 17.99$ & $0.0 \pm 50.39$ & 3.82 & 5.97 \\
\hline-8.92 & 13.69 & 16.34 & $1.04 \pm 0.15$ & $12.9 \pm 10.09$ & $39.49 \pm 16.63$ & 9.85 & 10.11 \\
\hline-7.74 & 13.53 & 15.59 & $1.1 \pm 0.12$ & $10.51 \pm 8.27$ & $52.87 \pm 12.05$ & 17.37 & 16.00 \\
\hline-6.53 & 13.37 & 14.88 & $1.09 \pm 0.11$ & $10.94 \pm 6.81$ & $49.15 \pm 10.36$ & 25.74 & 22.22 \\
\hline-5.29 & 13.21 & 14.23 & $1.13 \pm 0.1$ & $13.57 \pm 5.73$ & $43.8 \pm 9.32$ & 34.72 & 28.31 \\
\hline-4.02 & 13.04 & 13.65 & $1.2 \pm 0.1$ & $13.93 \pm 5.42$ & $45.91 \pm 8.63$ & 43.04 & 33.18 \\
\hline-2.73 & 12.88 & 13.17 & $1.28 \pm 0.1$ & $11.66 \pm 5.67$ & $55.92 \pm 8.17$ & 48.28 & 35.81 \\
\hline-1.41 & 12.72 & 12.79 & $1.36 \pm 0.11$ & $9.66 \pm 6.19$ & $67.75 \pm 8.19$ & 48.11 & 33.94 \\
\hline-0.07 & 12.55 & 12.55 & $1.45 \pm 0.12$ & $8.13 \pm 6.92$ & $76.22 \pm 8.75$ & 42.15 & 28.66 \\
\hline 1.29 & 12.39 & 12.45 & $1.54 \pm 0.14$ & $4.62 \pm 7.84$ & $83.56 \pm 9.58$ & 33.25 & 22.05 \\
\hline 2.67 & 12.22 & 12.51 & $1.55 \pm 0.15$ & $-1.29 \pm 8.73$ & $88.71 \pm 10.47$ & 24.17 & 15.88 \\
\hline 4.08 & 12.04 & 12.71 & $1.37 \pm 0.17$ & $1.85 \pm 9.63$ & $82.54 \pm 11.77$ & 15.74 & 10.82 \\
\hline 5.51 & 11.86 & 13.08 & $1.07 \pm 0.22$ & $12.55 \pm 13.75$ & $72.49 \pm 17.31$ & 8.84 & 7.30 \\
\hline-10.23 & 15.34 & 18.43 & $0.63 \pm 0.21$ & $-7.73 \pm 20.77$ & $0.0 \pm 54.64$ & 3.16 & 5.68 \\
\hline-9.08 & 15.18 & 17.69 & $1.01 \pm 0.15$ & $-3.23 \pm 10.25$ & $37.25 \pm 17.31$ & 9.82 & 9.87 \\
\hline-7.90 & 15.02 & 16.97 & $1.12 \pm 0.12$ & $5.69 \pm 7.87$ & $50.63 \pm 11.73$ & 17.89 & 16.00 \\
\hline-6.69 & 14.86 & 16.30 & $1.07 \pm 0.1$ & $6.94 \pm 6.53$ & $47.59 \pm 10.12$ & 26.61 & 22.92 \\
\hline-5.45 & 14.70 & 15.68 & $1.1 \pm 0.09$ & $8.05 \pm 5.66$ & $45.55 \pm 9.02$ & 35.99 & 29.49 \\
\hline-4.19 & 14.54 & 15.13 & $1.18 \pm 0.09$ & $8.33 \pm 5.43$ & $48.23 \pm 8.43$ & 45.47 & 35.58 \\
\hline-2.89 & 14.38 & 14.67 & $1.26 \pm 0.1$ & $8.46 \pm 5.74$ & $56.56 \pm 8.22$ & 52.88 & 39.36 \\
\hline-1.57 & 14.22 & 14.31 & $1.37 \pm 0.11$ & $7.95 \pm 6.06$ & $66.1 \pm 8.11$ & 55.52 & 39.30 \\
\hline-0.23 & 14.07 & 14.07 & $1.48 \pm 0.11$ & $5.96 \pm 6.4$ & $72.8 \pm 8.24$ & 51.37 & 35.12 \\
\hline 1.13 & 13.91 & 13.95 & $1.53 \pm 0.12$ & $3.05 \pm 6.66$ & $76.56 \pm 8.42$ & 41.59 & 28.01 \\
\hline 2.51 & 13.75 & 13.97 & $1.53 \pm 0.12$ & $1.32 \pm 6.84$ & $76.84 \pm 8.64$ & 30.52 & 20.58 \\
\hline 3.90 & 13.58 & 14.13 & $1.42 \pm 0.13$ & $2.8 \pm 7.42$ & $73.68 \pm 9.49$ & 20.61 & 14.20 \\
\hline 5.33 & 13.40 & 14.42 & $1.24 \pm 0.16$ & $9.27 \pm 9.1$ & $72.26 \pm 11.63$ & 13.03 & 9.44 \\
\hline 6.78 & 13.21 & 14.85 & $1.14 \pm 0.24$ & $20.12 \pm 14.12$ & $80.54 \pm 17.14$ & 7.88 & 6.03 \\
\hline-9.24 & 16.65 & 19.04 & $1.08 \pm 0.17$ & $-6.97 \pm 12.29$ & $47.19 \pm 18.37$ & 8.44 & 8.13 \\
\hline-8.06 & 16.49 & 18.35 & $1.17 \pm 0.13$ & $5.27 \pm 8.51$ & $52.77 \pm 12.39$ & 15.31 & 13.12 \\
\hline-6.85 & 16.33 & 17.71 & $1.04 \pm 0.11$ & $4.34 \pm 6.99$ & $49.17 \pm 10.62$ & 21.76 & 18.88 \\
\hline-5.61 & 16.17 & 17.11 & $1.04 \pm 0.09$ & $4.25 \pm 6.05$ & $45.84 \pm 9.58$ & 29.02 & 25.05 \\
\hline-4.34 & 16.01 & 16.59 & $1.12 \pm 0.09$ & $6.71 \pm 5.53$ & $45.17 \pm 8.87$ & 37.76 & 30.84 \\
\hline-3.05 & 15.85 & 16.14 & $1.22 \pm 0.1$ & $8.76 \pm 5.57$ & $50.74 \pm 8.42$ & 46.35 & 35.46 \\
\hline-1.72 & 15.70 & 15.79 & $1.34 \pm 0.1$ & $7.06 \pm 5.73$ & $58.39 \pm 8.1$ & 51.99 & 37.62 \\
\hline-0.38 & 15.55 & 15.56 & $1.42 \pm 0.11$ & $2.97 \pm 5.92$ & $64.46 \pm 8.02$ & 51.12 & 35.94 \\
\hline 0.98 & 15.41 & 15.44 & $1.46 \pm 0.11$ & $0.8 \pm 5.95$ & $67.79 \pm 7.89$ & 43.95 & 30.57 \\
\hline 2.35 & 15.26 & 15.44 & $1.48 \pm 0.11$ & $0.75 \pm 5.96$ & $68.94 \pm 7.86$ & 33.91 & 23.54 \\
\hline 3.73 & 15.10 & 15.55 & $1.41 \pm 0.11$ & $0.56 \pm 6.54$ & $69.77 \pm 8.56$ & 23.45 & 16.50 \\
\hline 5.14 & 14.92 & 15.78 & $1.28 \pm 0.14$ & $6.75 \pm 7.83$ & $70.51 \pm 10.13$ & 15.17 & 10.92 \\
\hline 6.59 & 14.72 & 16.13 & $1.21 \pm 0.19$ & $21.41 \pm 11.29$ & $70.78 \pm 14.42$ & 9.33 & 7.02 \\
\hline-9.39 & 18.09 & 20.38 & $1.03 \pm 0.25$ & $-9.87 \pm 16.49$ & $41.68 \pm 25.21$ & 6.03 & 5.46 \\
\hline-8.22 & 17.93 & 19.73 & $1.07 \pm 0.18$ & $-1.6 \pm 11.92$ & $48.69 \pm 17.62$ & 10.17 & 8.60 \\
\hline-7.02 & 17.77 & 19.10 & $0.89 \pm 0.13$ & $-1.24 \pm 9.54$ & $45.67 \pm 14.73$ & 13.22 & 12.61 \\
\hline-5.77 & 17.61 & 18.53 & $0.9 \pm 0.1$ & $0.82 \pm 7.19$ & $37.89 \pm 12.44$ & 17.64 & 17.08 \\
\hline-4.50 & 17.45 & 18.02 & $1.03 \pm 0.09$ & $6.67 \pm 5.77$ & $36.56 \pm 10.35$ & 25.09 & 21.86 \\
\hline-3.20 & 17.29 & 17.59 & $1.16 \pm 0.09$ & $9.68 \pm 5.42$ & $41.41 \pm 9.12$ & 33.54 & 26.56 \\
\hline-1.87 & 17.15 & 17.25 & $1.27 \pm 0.1$ & $6.2 \pm 5.44$ & $47.57 \pm 8.51$ & 40.25 & 30.15 \\
\hline-0.52 & 17.01 & 17.02 & $1.34 \pm 0.1$ & $0.49 \pm 5.47$ & $55.42 \pm 7.93$ & 43.06 & 31.31 \\
\hline 0.84 & 16.88 & 16.90 & $1.39 \pm 0.1$ & $-1.29 \pm 5.43$ & $60.96 \pm 7.54$ & 41.08 & 28.89 \\
\hline 2.20 & 16.76 & 16.90 & $1.4 \pm 0.1$ & $-1.23 \pm 5.39$ & $60.85 \pm 7.49$ & 34.11 & 23.45 \\
\hline 3.55 & 16.61 & 16.98 & $1.34 \pm 0.11$ & $-1.01 \pm 5.87$ & $58.92 \pm 8.26$ & 24.30 & 17.08 \\
\hline 4.94 & 16.41 & 17.14 & $1.23 \pm 0.14$ & $4.36 \pm 7.7$ & $60.09 \pm 10.65$ & 15.15 & 11.42 \\
\hline 6.39 & 16.20 & 17.42 & $1.09 \pm 0.19$ & $16.02 \pm 12.13$ & $60.18 \pm 16.41$ & 8.09 & 7.16 \\
\hline-7.18 & 19.19 & 20.48 & $0.64 \pm 0.19$ & $-15.47 \pm 15.25$ & $26.98 \pm 27.96$ & 6.26 & 7.04 \\
\hline-5.94 & 19.02 & 19.93 & $0.73 \pm 0.14$ & $-7.14 \pm 10.23$ & $18.22 \pm 23.65$ & 8.97 & 9.89 \\
\hline-4.66 & 18.86 & 19.43 & $0.92 \pm 0.11$ & $2.88 \pm 6.73$ & $22.86 \pm 15.28$ & 14.56 & 13.24 \\
\hline-3.35 & 18.70 & 19.00 & $1.1 \pm 0.11$ & $7.14 \pm 6.1$ & $33.01 \pm 11.53$ & 21.01 & 17.18 \\
\hline-2.01 & 18.55 & 18.66 & $1.21 \pm 0.1$ & $3.78 \pm 5.89$ & $40.66 \pm 9.97$ & 26.95 & 21.35 \\
\hline-0.65 & 18.42 & 18.43 & $1.29 \pm 0.1$ & $-2.28 \pm 5.37$ & $50.67 \pm 8.15$ & 32.17 & 24.13 \\
\hline 0.73 & 18.30 & 18.32 & $1.33 \pm 0.1$ & $-4.87 \pm 5.13$ & $55.22 \pm 7.47$ & 34.09 & 24.01 \\
\hline
\end{tabular}


Table 5

(Continued)

\begin{tabular}{|c|c|c|c|c|c|c|c|}
\hline $\begin{array}{l}\Delta \alpha^{\mathrm{a}} \\
(\mathrm{kpc})\end{array}$ & $\begin{array}{c}\Delta \delta^{\mathrm{a}} \\
(\mathrm{kpc})\end{array}$ & $\begin{array}{c}D^{\mathrm{b}} \\
(\mathrm{kpc})\end{array}$ & $\begin{array}{l}W_{0}^{\mathrm{c}} \\
(\AA)\end{array}$ & $\begin{array}{c}v^{\mathrm{d}} \\
\left(\mathrm{km} \mathrm{s}^{-1}\right)\end{array}$ & $\begin{array}{c}\sigma^{\mathrm{e}} \\
\left(\mathrm{km} \mathrm{s}^{-1}\right)\end{array}$ & $\Sigma_{\mathrm{Mg}}^{\mathrm{f}}$ & $\mathrm{S} / \mathrm{N}^{\mathrm{g}}$ \\
\hline 2.09 & 18.22 & 18.34 & $1.29 \pm 0.1$ & $-3.66 \pm 5.09$ & $49.4 \pm 7.84$ & 30.03 & 20.77 \\
\hline 3.37 & 18.10 & 18.41 & $1.21 \pm 0.11$ & $-1.2 \pm 5.53$ & $41.76 \pm 9.29$ & 21.89 & 15.61 \\
\hline 4.71 & 17.86 & 18.47 & $1.15 \pm 0.15$ & $0.9 \pm 7.81$ & $42.9 \pm 12.68$ & 13.27 & 10.54 \\
\hline 6.17 & 17.63 & 18.68 & $0.91 \pm 0.21$ & $1.42 \pm 15.36$ & $44.07 \pm 23.2$ & 5.92 & 6.50 \\
\hline-6.10 & 20.41 & 21.30 & $0.71 \pm 0.24$ & $-12.52 \pm 18.58$ & $29.37 \pm 31.88$ & 4.39 & 5.24 \\
\hline-4.82 & 20.25 & 20.81 & $0.84 \pm 0.16$ & $-0.98 \pm 10.3$ & $20.66 \pm 22.64$ & 7.98 & 7.44 \\
\hline-3.51 & 20.08 & 20.39 & $1.02 \pm 0.13$ & $-2.73 \pm 7.83$ & $24.41 \pm 16.81$ & 12.04 & 10.64 \\
\hline-2.16 & 19.92 & 20.04 & $1.16 \pm 0.11$ & $-6.59 \pm 6.55$ & $34.32 \pm 12.06$ & 16.93 & 14.25 \\
\hline-0.79 & 19.76 & 19.78 & $1.24 \pm 0.1$ & $-10.46 \pm 5.74$ & $43.69 \pm 9.37$ & 22.00 & 17.31 \\
\hline 0.62 & 19.61 & 19.62 & $1.24 \pm 0.1$ & $-10.93 \pm 5.77$ & $47.87 \pm 8.98$ & 24.23 & 18.27 \\
\hline 2.05 & 19.48 & 19.59 & $1.17 \pm 0.11$ & $-6.19 \pm 6.22$ & $43.38 \pm 10.15$ & 21.74 & 16.45 \\
\hline 3.02 & 19.42 & 19.65 & $1.11 \pm 0.13$ & $0.25 \pm 6.74$ & $33.76 \pm 12.55$ & 16.22 & 12.96 \\
\hline 4.47 & 19.13 & 19.65 & $1.14 \pm 0.17$ & $3.47 \pm 9.36$ & $35.25 \pm 16.5$ & 10.24 & 8.83 \\
\hline 5.99 & 18.97 & 19.89 & $0.99 \pm 0.25$ & $-3.41 \pm 18.06$ & $38.75 \pm 28.3$ & 4.52 & 5.52 \\
\hline-3.68 & 21.44 & 21.75 & $0.84 \pm 0.19$ & $-19.9 \pm 12.44$ & $0.0 \pm 41.13$ & 6.07 & 6.44 \\
\hline-2.33 & 21.27 & 21.39 & $1.03 \pm 0.14$ & $-16.25 \pm 7.86$ & $8.07 \pm 25.2$ & 10.01 & 9.20 \\
\hline-0.96 & 21.08 & 21.10 & $1.1 \pm 0.13$ & $-13.5 \pm 7.23$ & $26.17 \pm 15.19$ & 13.68 & 11.82 \\
\hline 0.43 & 20.88 & 20.88 & $1.07 \pm 0.13$ & $-12.43 \pm 8.24$ & $40.84 \pm 13.59$ & 14.73 & 12.94 \\
\hline 1.79 & 20.63 & 20.71 & $1.04 \pm 0.14$ & $-7.69 \pm 10.08$ & $54.91 \pm 14.34$ & 12.85 & 12.31 \\
\hline 3.04 & 20.43 & 20.65 & $1.06 \pm 0.15$ & $5.35 \pm 9.8$ & $42.89 \pm 15.59$ & 9.92 & 9.87 \\
\hline 4.39 & 20.38 & 20.84 & $1.22 \pm 0.19$ & $10.09 \pm 12.05$ & $38.32 \pm 19.77$ & 7.03 & 6.91 \\
\hline-2.52 & 22.61 & 22.75 & $0.74 \pm 0.22$ & $-13.84 \pm 17.95$ & $0.0 \pm 50.39$ & 4.02 & 5.75 \\
\hline-1.16 & 22.42 & 22.45 & $0.89 \pm 0.18$ & $-1.3 \pm 12.27$ & $0.0 \pm 40.9$ & 6.70 & 7.75 \\
\hline 0.21 & 22.23 & 22.23 & $0.84 \pm 0.17$ & $-1.64 \pm 14.06$ & $36.13 \pm 23.24$ & 7.27 & 8.78 \\
\hline 1.57 & 22.03 & 22.09 & $0.82 \pm 0.2$ & $-0.63 \pm 20.76$ & $67.59 \pm 26.42$ & 5.85 & 8.35 \\
\hline 2.92 & 21.88 & 22.07 & $0.88 \pm 0.19$ & $11.57 \pm 16.88$ & $37.77 \pm 26.74$ & 4.52 & 6.80 \\
\hline 0.03 & 23.62 & 23.62 & $0.6 \pm 0.25$ & $1.75 \pm 28.58$ & $6.44 \pm 60.09$ & 2.00 & 5.22 \\
\hline 1.40 & 23.46 & 23.50 & $0.47 \pm 0.27$ & $-11.35 \pm 40.72$ & $0.0 \pm 81.57$ & $\ldots$ & 5.02 \\
\hline
\end{tabular}

Notes. Nonphysical Mg II absorption significance values are left blank.

a Arc-position physical separation to galaxy in the absorber plane.

${ }^{\mathrm{b}}$ Projected physical separation to galaxy in the absorber plane.

${ }^{\mathrm{c}} \mathrm{Mg}$ II $\lambda 2796$ absorption strength (with $1 \sigma$ error).

${ }^{\mathrm{d}}$ Velocity offset relative to $z=0.7711$ (with $1 \sigma$ error).

${ }^{\mathrm{e}}$ Velocity dispersion relative to $z=0.7711$ (with $1 \sigma$ error).

${ }^{\mathrm{f}} \mathrm{Mg}$ II absorption significance values.

${ }^{\mathrm{g}} \mathrm{S} / \mathrm{N}$ to the continuum.

underlying these processes, we probed the cool metal-enriched CGM of a $z=0.77$ star-forming galaxy (within the CSWA 38 lens system) through a tomography technique using bright giant gravitational arcs as background sources. This adds to the currently small sample of galaxies whose CGM has been mapped spatially in absorption using integral field spectroscopy combined with gravitational lensing, a technique pioneered by Lopez et al. (2018).

Our study is based on observations obtained with the KCWI. We have measured Mg II $\lambda \lambda 2796,2803$ equivalent widths $\left(W_{0}\right)$, velocity offsets $(v)$, and velocity dispersions $(\sigma)$ in a total of 280 spaxels, each corresponding to $\simeq 2 \mathrm{kpc}^{2}$ at $z=0.77$ for a typical magnification factor $\mu \simeq 3$. The spatial resolution is 1 !" 0 FWHM in the image plane and $\simeq 15 \mathrm{kpc}^{2}$ at $z=0.77$. This configuration has allowed us to probe the CGM in an individual galactic environment at impact parameters $D \simeq 5-30 \mathrm{kpc}$, in addition to the absorber galaxy using downthe-barrel spectroscopy $(D=0)$. Our findings can be summarized as follows.

1. The CGM gas is well detected in Mg II absorption against both background arcs out to $D \approx 25 \mathrm{kpc}$. Spatial variation in absorption equivalent widths combined with absorption line ratios indicates an optically thick medium with patchy distribution (i.e., varying covering fraction). These results are broadly consistent with the clumpy CGM inferred from previous tomographic CGM measurements using gravitational lensing (Lopez et al. 2018, 2020).

2. We observe a $W_{0}-D$ anticorrelation in the $\mathrm{Mg}$ II absorption. Both arcs in the CSWA 38 lens system lie near the mean of a fit to the Nielsen et al. (2013a) data, and the scatter of $W_{0}^{2796}$ in both arcs (Figure 9) is far smaller than that measured from quasar sight lines through different halos. This result also holds for the two other systems with similar lens tomography measurements (Lopez et al. 2018, 2020). We attribute the relatively small scatter within the lens tomography measurements to a combination of (1) "halo-to-halo" variation in the CGM around different galaxies and (2) small-scale "intrahalo" variations within the CGM of individual galaxies. The latter point is especially interesting, as it probes the size scale of absorbing clouds within the CGM. Since the arc tomography presented here effectively averages over areas of $\sim 15 \mathrm{kpc}^{2}$ (i.e., 
much larger than for QSOs), the small scatter in the $W_{0}-D$ relation suggests that $\mathrm{Mg}$ II absorption in the CGM arises from individual components of $\lesssim \mathrm{kpc}$ size, in qualitative agreement with intrahalo variations seen toward lensed QSO sight lines.

3. Absorption line kinematics in the arcs suggest that the Mg II-bearing gas is largely dispersion-supported in the regions probed, in contrast to other systems, which show a higher degree of rotational support (Lopez et al. $2018,2020)$. The velocity dispersion is at least half of the expected rotation velocity. The absorber galaxy spectrum shows clear outflow kinematics in down-the-barrel $\mathrm{Mg}$ II absorption, while evidence of a subdued outflow component is prevalent in one of the background arc sight lines (arc 2) and detected over $D \approx 10-20 \mathrm{kpc}$.

Future study of the CSWA 38 lens system will benefit from an accurate measurement of the absorber galaxy rotation curve, for example, from nebular emission lines such as [O II] or $\mathrm{H} \alpha$. This will either help confirm the dispersion-dominated interpretation or identify a more significant rotation component. Regardless, our study demonstrates that tomographic mapping continues to provide new, more detailed insights into the structure and kinematics of the CGM. This technique thus adds considerably to our toolkit for understanding the gas inflow/outflow processes that regulate star formation and quenching in evolving galaxies.

This work is based on data obtained at the W. M. Keck Observatory, which is operated as a scientific partnership among the California Institute of Technology, the University of California, and the National Aeronautics and Space Administration. The Observatory was made possible by the generous financial support of the W. M. Keck Foundation. We wish to acknowledge the very significant cultural role and reverence that the summit of Maunakea has within the indigenous Hawaiian community. We are most fortunate to have the opportunity to conduct observations from this sacred mountain, and we respectfully say mahalo. K.M. acknowledges support for this work from the National Science Foundation (NSF) under grant PHY-1852581 and the Dean's Distinguished Graduate Fellowship, funded by the Office of Graduate Studies and the Dean of the College of Letters and Science at the University of California, Davis. T.J. and K.V.G.C. acknowledge support from the Gordon and Betty Moore Foundation through grant GBMF8549. C.A.F. G. was supported by the NSF through grant AST-1715216 and CAREER award AST-1652522; NASA through grant 17-ATP170067; STScI through grants HST-GO-14681.011, HST-GO14268.022-A, HST-AR-14293.001-A, and HST-AR-16124.001A; and a Cottrell Scholar Award from the Research Corporation for Science Advancement. We thank the anonymous referee for a careful and constructive report that has improved this manuscript.

Facility: Keck (KCWI).

\section{ORCID iDs}

Kris Mortensen (iD https://orcid.org/0000-0001-9676-5005 Tucker Jones (i) https://orcid.org/0000-0001-5860-3419 Claude-André Faucher-Giguère (iD https://orcid.org/00000002-4900-6628

Ryan L. Sanders (iD https://orcid.org/0000-0003-4792-9119

Richard S. Ellis (i) https://orcid.org/0000-0001-7782-7071

Nicha Leethochawalit (i) https://orcid.org/0000-0003-

4570-3159

\section{References}

Anglés-Alcázar, D., Faucher-Giguère, C.-A., Quataert, E., et al. 2017, MNRAS, 472, L109

Bayliss, M. B., Hennawi, J. F., Gladders, M. D., et al. 2011, ApJS, 193, 8 Behroozi, P. S., Wechsler, R. H., \& Conroy, C. 2013, ApJ, 770, 57

Belokurov, V., Evans, N. W., Hewett, P. C., et al. 2009, MNRAS, 392, 104 Belokurov, V., Evans, N. W., Moiseev, A., et al. 2007, ApJL, 671, L9

Bordoloi, R., Lilly, S. J., Kacprzak, G. G., \& Churchill, C. W. 2014, ApJ, 784, 108

Bordoloi, R., Lilly, S. J., Knobel, C., et al. 2011, ApJ, 743, 10

Bouché, N., Hohensee, W., Vargas, R., et al. 2012, MNRAS, 426, 801

Bruzual, G., \& Charlot, S. 2003, MNRAS, 344, 1000

Bullock, J. S., \& Boylan-Kolchin, M. 2017, ARA\&A, 55, 343

Burchett, J. N., Rubin, K. H. R., Prochaska, J. X., et al. 2021, ApJ, 909, 151

Burkert, A., Genzel, R., Bouché, N., et al. 2010, ApJ, 725, 2324

Calzetti, D., Armus, L., Bohlin, R. C., et al. 2000, ApJ, 533, 682

Chambers, K. C., Magnier, E. A., Metcalfe, N., et al. 2016, arXiv:1612.05560

Chen, H.-W. 2017, in Outskirts of Galaxies, Vol. 434, ed. J. H. Knapen, J. C. Lee, \& A. Gil de Paz (Berlin: Springer), 291

Chen, H.-W., Gauthier, J.-R., Sharon, K., et al. 2014, MNRAS, 438, 1435

Chen, H.-W., Helsby, J. E., Gauthier, J.-R., et al. 2010, ApJ, 714, 1521

Dai, L., Kaurov, A. A., Sharon, K., et al. 2020, MNRAS, 495, 3192

Dekel, A., Birnboim, Y., Engel, G., et al. 2009, Natur, 457, 451

Diamond-Stanic, A. M., Coil, A. L., Moustakas, J., et al. 2016, ApJ, 824, 24

Elahi, P. J., Power, C., Lagos, C. d. P., Poulton, R., \& Robotham, A. S. G. 2018, MNRAS, 477, 616

Ellison, S. L., Ibata, R., Pettini, M., et al. 2004, A\&A, 414, 79

Hafen, Z., Faucher-Giguère, C.-A., Anglés-Alcázar, D., et al. 2017, MNRAS, 469, 2292

Hafen, Z., Faucher-Giguere, C. A., Angles-Alcazar, D., et al. 2020, MNRAS, 494, 3581

Hafen, Z., Faucher-Giguère, C.-A., Anglés-Alcázar, D., et al. 2019b, MNRAS, 488, 1248

Ho, S. H., Martin, C. L., Kacprzak, G. G., \& Churchill, C. W. 2017, ApJ, 835,267

Ilbert, O., Arnouts, S., McCracken, H. J., et al. 2006, A\&A, 457, 841

Jones, T., Stark, D. P., \& Ellis, R. S. 2018, ApJ, 863, 191

Kacprzak, G. G., Churchill, C. W., \& Nielsen, N. M. 2012, ApJL, 760, L7

Kereš, D., Katz, N., Weinberg, D. H., \& Davé, R. 2005, MNRAS, 363, 2

Koester, B. P., Gladders, M. D., Hennawi, J. F., et al. 2010, ApJL, 723, L73

Kornei, K. A., Shapley, A. E., Martin, C. L., et al. 2013, ApJ, 774, 50

Kriek, M., van Dokkum, P. G., Labbé, I., et al. 2009, ApJ, 700, 221

Lan, T.-W., Ménard, B., \& Zhu, G. 2014, ApJ, 795, 31

Lan, T.-W., \& Mo, H. 2018, ApJ, 866, 36

Lehner, N., Berek, S. C., Howk, J. C., et al. 2020, ApJ, 900, 9

Lehner, N., Howk, J. C., Tripp, T. M., et al. 2013, ApJ, 770, 138

Lopez, S., Ellison, S., D’Odorico, S., \& Kim, T. S. 2007, A\&A, 469, 61

Lopez, S., Reimers, D., Gregg, M. D., et al. 2005, ApJ, 626, 767

Lopez, S., Reimers, D., Rauch, M., Sargent, W. L. W., \& Smette, A. 1999, ApJ, 513, 598

Lopez, S., Tejos, N., Barrientos, L. F., et al. 2020, MNRAS, 491, 4442

Lopez, S., Tejos, N., Ledoux, C., et al. 2018, Natur, 554, 493

Martin, C. L., Ho, S. H., Kacprzak, G. G., \& Churchill, C. W. 2019, ApJ, 878,84

Morrissey, P., Matuszewski, M., Martin, D. C., et al. 2018, ApJ, 864, 93

Navarro, J. F., Frenk, C. S., \& White, S. D. M. 1997, ApJ, 490, 493

Nielsen, N. M., Churchill, C. W., \& Kacprzak, G. G. 2013a, ApJ, 776, 115

Nielsen, N. M., Churchill, C. W., Kacprzak, G. G., \& Murphy, M. T. 2013b, ApJ, 776, 114

Oguri, M. 2010, PASJ, 62, 1017

Oppenheimer, B. D., Davé, R., Kereš, D., et al. 2010, MNRAS, 406, 2325

Prochaska, J. X., Werk, J. K., Worseck, G., et al. 2017, ApJ, 837, 169

Rauch, M., Sargent, W. L. W., Barlow, T. A., \& Carswell, R. F. 2001, ApJ, 562,76

Rubin, K. H. R., Diamond-Stanic, A. M., Coil, A. L., Crighton, N. H. M., \& Moustakas, J. 2018a, ApJ, 853, 95

Rubin, K. H. R., Diamond-Stanic, A. M., Coil, A. L., Crighton, N. H. M., \& Stewart, K. R. 2018b, ApJ, 868, 142

Rubin, K. H. R., O’Meara, J. M., Cooksey, K. L., et al. 2018c, ApJ, 859, 146

Rubin, K. H. R., Prochaska, J. X., Koo, D. C., et al. 2014, ApJ, 794, 156

Rudie, G. C., Steidel, C. C., Trainor, R. F., et al. 2012, ApJ, 750, 67

Schroetter, I., Bouché, N., Wendt, M., et al. 2016, ApJ, 833, 39

Shull, J. M. 2014, ApJ, 784, 142

Smette, A., Surdej, J., Shaver, P. A., et al. 1992, ApJ, 389, 39

Sommer-Larsen, J. 1991, MNRAS, 249, 368 
Steidel, C. C., Erb, D. K., Shapley, A. E., et al. 2010, ApJ, 717, 289

Stern, J., Faucher-Giguère, C.-A., Fielding, D., et al. 2020, arXiv:2006.13976

Tumlinson, J., Peeples, M. S., \& Werk, J. K. 2017, ARA\&A, 55, 389

van de Voort, F., Schaye, J., Booth, C. M., \& Dalla Vecchia, C. 2011, MNRAS, 415, 2782

van den Bergh, S. 1962, AJ, 67, 486

Veilleux, S., Cecil, G., \& Bland-Hawthorn, J. 2005, ARA\&A, 43, 769
Wellons, S., Faucher-Giguère, C.-A., Anglés-Alcázar, D., et al. 2020, MNRAS, 497, 4051

Whitaker, K. E., Franx, M., Leja, J., et al. 2014, ApJ, 795, 104

Woolf, V. M., \& West, A. A. 2012, MNRAS, 422, 1489

Zabl, J., Bouché, N. F., Schroetter, I., et al. 2020, MNRAS, 492, 4576

Zahedy, F. S., Chen, H.-W., Rauch, M., Wilson, M. L., \& Zabludoff, A. 2016, MNRAS, 458, 2423 